\title{
ON THE SPECTRUM OF AN ANHARMONIC OSCILLATOR
}

\author{
MARCO MUGHETTI
}

\begin{abstract}
We consider the one-dimensional anharmonic oscillator $-\partial_{x}^{2}+$ $a x^{2 h}+\mu x^{h-1}$ and we study the qualitative behaviour of its eigenvalues $\lambda_{j}$; in particular, we show how the sign of its eigenvalues depends on the parameters $h \in \mathbb{N}, a \in \mathbb{R}_{+}, \mu \in \mathbb{R}$. We applied our results to the study of $C^{\infty}$ hypoellipticity and of a priori estimates for certain non-transversally elliptic operators.
\end{abstract}

\section{INTRODUCTION}

In this paper we study the one-dimensional anharmonic oscillator

$$
P_{\mu}=-\partial_{x}^{2}+a x^{2 h}+\mu x^{h-1},
$$

where $h \in \mathbb{N}, a \in \mathbb{R}_{+}$are fixed positive constants and $\mu \in \mathbb{R}$ is a parameter. The operator $P_{\mu}$ is a perturbation of the anharmonic oscillator $-\partial_{x}^{2}+a x^{2 h}$, and its lowest degree term $x^{h-1}$ is chosen in such a way to behave as the commutator $\left[\partial_{x}, \sqrt{a} x^{h}\right]$. The spectral properties of the operator $P_{\mu}$ arise naturally in many questions (see, for instance, Gilioli-Treves [11, Menikoff [26], Hanges-Himonas 14], Cordaro-Hanges [9], Kohn [24], Mughetti-Parenti-Parmeggiani [31]).

Recently Helffer in [18 and Helffer-Persson in 19 have carried out a sharp analysis of the ground state energy associated with the anharmonic oscillator $Q_{\alpha}=$ $-\partial_{x}^{2}+\left(x^{h} / h-\alpha\right)^{2}, \alpha \in \mathbb{R}$. As we shall see in Section 3 , although the potentials in $Q_{\alpha}$ and in $P_{\mu}$ are very similar, the corresponding lowest eigenvalues behave in a somewhat different way. Some remarks are in order.

The oscillator $P_{\mu}$, thought as an $L^{2}(\mathbb{R})$-unbounded operator on the domain

$$
B_{h}=\left\{f \in L^{2}(\mathbb{R}),\|f\|_{B_{h}}=\left(\sum_{\alpha / h+\beta \leq 2}\left\|x^{\alpha} \partial_{x}^{\beta} f\right\|_{L^{2}(\mathbb{R})}^{2}\right)^{\frac{1}{2}}<+\infty\right\},
$$

turns out to be self-adjoint. Moreover, due to $a>0$, the potential $V(x)=a x^{2 h}+$ $\mu x^{h-1} \underset{|x| \rightarrow+\infty}{\longrightarrow}+\infty$ and by Theorem XIII.67 [32] (see also Theorem 3.1, Chap. 2 in [1]) we have that $P_{\mu}$ has a purely discrete spectrum, i.e., $\operatorname{Spec}\left(P_{\mu}\right)$ is made of isolated eigenvalues $\lambda_{j}(\mu), j \in \mathbb{N}$, of finite multiplicities, diverging to $+\infty$. Actually, by Proposition 3.3, Chap. 2 in [1] every eigenvalue $\lambda_{j}(\mu)$ is simple (i.e., of multiplicity one). We can arrange the spectrum of $P_{\mu}$ in such a way that, for every $\mu \in \mathbb{R}$,

$$
\lambda_{0}(\mu)<\lambda_{1}(\mu)<\cdots<\lambda_{j}(\mu)<\lambda_{j+1}(\mu)<\cdots, \quad j \in \mathbb{N} .
$$

Received by the editors December 29, 2011 and, in revised form, June 15, 2012.

2010 Mathematics Subject Classification. Primary 34L15.

Key words and phrases. Anharmonic oscillator, pseudodifferential operators, eigenvalues, a priori estimates, hypoellipticity. 
Furthermore, as shown in Appendix I, $\left\{P_{\mu}\right\}_{\mu \in \mathbb{R}}$ is an analytic family of type $(A)$ and thus an analytic family in the sense of Kato (see Definitions in XII.2 of [32, pp. 14, 16). Therefore, in view of Theorem XII.8, the eigenvalues $\lambda_{j}(\mu)$ are analytic functions of $\mu \in \mathbb{R}$ (we refer to Appendix I for further details).

If $h=1, P_{\mu}$ reduces to the classical harmonic oscillator and its spectrum can be completely computed by using, for instance, the creation operator $L_{+}=-\partial_{x}+$ $\sqrt{a} x^{h}$; precisely, for $h=1$ we have

$$
\begin{gathered}
P_{\mu} e^{-\frac{\sqrt{a} x^{2}}{2}}=(\sqrt{a}+\mu) e^{-\frac{\sqrt{a} x^{2}}{2}}, \\
P_{\mu} L_{+}=L_{+} P_{\mu}+\left[P_{\mu}, L_{+}\right]=L_{+}\left(P_{\mu}+2 \sqrt{a}\right),
\end{gathered}
$$

whence it is easily seen that

$$
\lambda_{j}(\mu)=\sqrt{a}(2 j+1)+\mu,
$$

where the corresponding eigenfunction $\phi_{j}$ is the $j$-Hermite function

$$
\phi_{j}(x)=c_{j} L_{+}^{j} e^{-\frac{\sqrt{a} x^{2}}{2}}, \quad c_{j} \in \mathbb{C} .
$$

Here the computation of the eigenvalues rests on two key points: the lower degree term $\mu x^{h-1} \equiv \mu$ in $P_{\mu}$ is actually a shift-operator and the commutator $\left[P_{\mu}, L_{+}\right]$is proportional to $L_{+}$of a constant factor $2 \sqrt{a}$. Unfortunately, for any integer $h>1$ this is no longer true, and, to our knowledge, there is not any explicit description of the spectrum of $P_{\mu}$ in terms of the parameter $\mu$. Our aim is, firstly, to carry out a precise analysis of the sign of the eigenvalues $\lambda_{j}(\mu)$ as $\mu$ varies in $\mathbb{R}$; secondly, we shall apply this result to the study of the lower bounds and of the hypoellipticity of some non-transversally elliptic operators (i.e., classical pseudodifferential operators, whose principal symbol does not vanish exactly to the second order on its characteristic manifold). In particular we show a somewhat different behaviour of the spectrum of $P_{\mu}$ depending on the parity of $h$ (in this connection we also refer to Section 3 ).

According to (1.3), the main result of this section is the following

Theorem 1.1. If $h$ is a positive even integer, then we have, for any $j \in \mathbb{N}$,

$$
\left\{\begin{array}{lll}
\lambda_{j}(\mu)>0 & \text { if } & |\mu|<\sqrt{a}(h+1)(2 j+1) ; \\
\lambda_{j}(\mu)=0 & \text { if } & |\mu|=\sqrt{a}(h+1)(2 j+1) ; \\
\lambda_{j}(\mu)<0 & \text { if } & |\mu|>\sqrt{a}(h+1)(2 j+1) .
\end{array}\right.
$$

On the other hand, if $h$ is a positive odd integer, then we get, for any $j \in \mathbb{N}$,

$$
\begin{cases}\lambda_{j}(\mu)>0 & \text { if } \quad \mu>(-1)^{j} \sqrt{a}-\sqrt{a}(h+1)(j+\theta(j)) ; \\ \lambda_{j}(\mu)=0 & \text { if } \quad \mu=(-1)^{j} \sqrt{a}-\sqrt{a}(h+1)(j+\theta(j)) ; \\ \lambda_{j}(\mu)<0 & \text { if } \quad \mu<(-1)^{j} \sqrt{a}-\sqrt{a}(h+1)(j+\theta(j)),\end{cases}
$$

where, from now on, $\theta(j):=1$ if $j$ is even and $\theta(j):=0$ if $j$ is odd.

Furthermore, $P_{\mu}$ turns out to be an $L^{2}(\mathbb{R})$-non-negative operator (namely, $\left.\left\langle P_{\mu} u, u\right\rangle \geq C\|u\|_{L^{2}(\mathbb{R})}^{2}, C \geq 0\right)$ if and only if

$$
\mu \geq-h \sqrt{a} \text { if } h \text { is odd and } \quad|\mu| \leq(h+1) \sqrt{a} \text { if } h \text { is even. }
$$

Finally, $P_{\mu}$ is an $L^{2}(\mathbb{R})$-positive operator if and only if the inequalities (1.7) are strict. 
Note that, for $h=1$, one has $(-1)^{j} \sqrt{a}-\sqrt{a}(h+1)(j+\theta(j))=-\sqrt{a}(2 j+1)$; therefore conditions (1.6) easily follow from (1.4).

In the even case, the eigenvalues $\lambda_{j}(\mu)$ show a symmetry with respect to the origin, which is completely absent in the odd case. Indeed, only in the even case, the operators $P_{\mu}$ and $P_{-\mu}$ are unitarily equivalent, via the change of variable $x \rightarrow-x$. As a consequence, they have the same spectrum, and from (1.3) one easily gets that

$$
\lambda_{j}(-\mu)=\lambda_{j}(\mu) \quad \text { for every } \quad \mu \in \mathbb{R} \quad \text { and } j \in \mathbb{N} \quad(h \text { even }) .
$$

In [8], the authors consider the one-dimensional Schrödinger operator in $\mathbb{R}$,

$$
H=-\partial_{x}^{2}+V(x),
$$

and prove the following upper bound for the number $N_{-}(H)$ of the negative eigenvalues of $H$ (see (29) in [8]):

$$
N_{-}(H) \leq 1+\sqrt{2}\left[\int_{-\infty}^{+\infty} x^{2} V^{-}(x) d x \int_{-\infty}^{+\infty} V^{-}(x) d x\right]^{1 / 4},
$$

where $V^{-}(x)=-\min \{V(x), 0\}$. We are now going to use Theorem 1.1 to check the sharpness of the bound (1.9), applied to our anharmonic oscillator $P_{\mu}$ in (1.1). To see this, fix

$$
\mu_{\ell}=-\sqrt{a}(h+1)(2 \ell+1)
$$

for some $\ell \in \mathbb{N}$ and, to begin with, assume that $h$ is even. Then it follows that $V(x)=a x^{2 h}+\mu_{\ell} x^{h-1} \leq 0$ whenever $0 \leq x \leq \sigma:=\sqrt[h+1]{-\mu_{\ell} / a}$, whence one gets

$$
\begin{gathered}
\int_{-\infty}^{+\infty} x^{2} V^{-}(x) d x=\int_{0}^{\sigma}\left(-a x^{2 h+2}-\mu_{\ell} x^{h+1}\right) d x=\frac{\sigma \mu_{\ell}^{2}}{a} \frac{h+1}{(h+2)(2 h+3)}, \\
\int_{-\infty}^{+\infty} V^{-}(x) d x=\int_{0}^{\sigma}\left(-a x^{2 h}-\mu_{\ell} x^{h-1}\right) d x=\frac{\mu_{\ell}^{2}}{\sigma a} \frac{h+1}{(2 h+1) h} .
\end{gathered}
$$

Thus, in view of (1.10), the upper bound of $N_{-}\left(P_{\mu_{\ell}}\right)$ in (1.9) turns out to be

$$
1+\sqrt{2}(2 \ell+1)(h+1)\left[\frac{(h+1)^{2}}{h(h+2)(2 h+1)(2 h+3)}\right]^{1 / 4} \simeq \sqrt{h}(2 \ell+1), \quad \text { if } h \gg 1 .
$$

On the other hand, due to Theorem 1.1, we have that $N_{-}\left(P_{\mu_{\ell}}\right)=\ell$; this shows that (1.9) provides a quite good estimate for the growth of $N_{-}\left(P_{\mu_{\ell}}\right)$, although the factor $\sqrt{h}$ can be somewhat large.

In the case of odd $h$, the argument leads to the same conclusion. Precisely, we easily see that $V(x) \leq 0$ whenever $-\sigma \leq x \leq \sigma$ and hence, arguing as above, the right hand side of (1.9) is equal to

$$
1+\sqrt{2}(2 \ell+1)(h+1)\left[\frac{4(h+1)^{2}}{h(h+2)(2 h+1)(2 h+3)}\right]^{1 / 4} \simeq \sqrt{2 h}(2 \ell+1), \quad \text { if } h \gg 1 .
$$

Finally, again from Theorem 1.1, we have $N_{-}\left(P_{\mu_{\ell}}\right)=2 \ell+1$.

We end this section with a corollary of Theorem 1.1 about the structure of the eigenvalues $\lambda_{j}(\mu)$. 
Corollary 1.2. If $h$ is a positive integer, then, for any $j \in \mathbb{N}$, there exists an analytic function $\tilde{\lambda}_{j}$, with $\tilde{\lambda}_{j}(\mu)<0$ for $\mu \in \mathbb{R}$, such that

$$
\lambda_{j}(\mu)=\left\{\begin{array}{l}
\tilde{\lambda}_{j}(\mu)\left(\mu^{2}-a(h+1)^{2}(2 j+1)^{2}\right), \quad \text { if } h \text { is even } \\
\tilde{\lambda}_{j}(\mu)\left((-1)^{j} \sqrt{a}-\sqrt{a}(h+1)(j+\theta(j))-\mu\right), \quad \text { if } h \text { is odd. }
\end{array}\right.
$$

In the even case, $\tilde{\lambda}_{j}(\mu)$ turns out to be an even function.

The paper is organized as follows: in Section 2 we prove Theorem 1.1 and Corollary 1.2. in Section 3 we study the qualitative behaviour of the eigenvalues $\lambda_{j}(\mu)$; in Sections 4 and 5 we discuss two applications of Theorem 1.1 to lower bounds and to $C^{\infty}$-hypoellipticity of certain non-transversally elliptic pseudodifferential operators.

\section{Proof of Theorem 1.1 and of Corollary 1.2}

The first part of this section is devoted to the proof of Theorem 1.1. We start off by computing the set of values of $\mu$ for which $P_{\mu}$ is not $L^{2}$-injective, i.e., $\operatorname{Ker}_{L^{2}(\mathbb{R})} P_{\mu} \neq\langle 0\rangle$.

We have to distinguish two cases, according to the parity of $h$.

Assume firstly that $h$ is an odd integer and consider the following second order pseudodifferential operator in $\mathbb{R}_{(t, y)}^{2}$ :

$$
\begin{aligned}
P_{\text {odd }} & =-\partial_{t}^{2}-a t^{2 h} \partial_{y}^{2}+\mu t^{h-1}\left|D_{y}\right| \\
& =-\left(\partial_{t}-\sqrt{a} t^{h}\left|D_{y}\right|\right)\left(\partial_{t}+\sqrt{a} t^{h}\left|D_{y}\right|\right)+(\sqrt{a} h+\mu) t^{h-1}\left|D_{y}\right| .
\end{aligned}
$$

Here $\left|D_{y}\right|$ denotes a pseudodifferential operator with symbol $|\eta|$ microlocally near $\tau=0$. In view of the Main Theorem in [12] (see also [1] ), we find out that $P_{\text {odd }}$ is hypoelliptic (with loss of $2 h /(h+1)$ derivatives) if and only if

$$
-\frac{\mu+\sqrt{a} h}{2 \sqrt{a}} \neq j(h+1) \text { or } j(h+1)+1, \quad \forall j \in \mathbb{N} .
$$

On the other hand, in view of Theorem 1.12 in [27, $P_{\text {odd }}$ is hypoelliptic (with loss of $2 h /(h+1)$ derivatives) if and only if the associated localized operator $-\partial_{t}^{2}+$ $a t^{2 h} \eta^{2}+\mu t^{h-1}|\eta|$ is injective in $L^{2}(\mathbb{R})$ (or, equivalently, in $\mathcal{S}(\mathbb{R})$ ), for every $\eta \neq 0$. By performing the change of variable $x=|\eta|^{\frac{1}{h+1}} t$, the above condition amounts to saying that

$$
P_{\mu}=-\partial_{x}^{2}+a x^{2 h}+\mu x^{h-1} \text { is injective in } L^{2}(\mathbb{R}) .
$$

In order to treat the even case, now consider the following second order differential operator in $R_{(x, y)}^{2}$ (with $\left.D=\frac{1}{\sqrt{-1}} \partial\right)$ :

$$
\begin{aligned}
P_{\text {even }} & =D_{x}^{2}+a x^{2 h} D_{y}^{2}+\mu x^{h-1} D_{y} \\
& =\left(D_{x}-i \sqrt{a} x^{h} D_{y}\right)\left(D_{x}+i \sqrt{a} x^{h} D_{y}\right)+(\mu-\sqrt{a} h) x^{h-1} D_{y} .
\end{aligned}
$$

Due to Theorem 2 in [26], $P_{\text {even }}$ is hypoelliptic (with loss of $2 h /(h+1)$ derivatives) if and only if

$$
\frac{\mu-\sqrt{a} h}{2 \sqrt{a}} \neq j(h+1)+1 / 2, \quad \forall j \in \mathbb{Z} .
$$


Again, by virtue of Theorem 1.12 [27], this holds if and only if the associated localized operator

$$
D_{x}^{2}+a x^{2 h} \eta^{2}+\mu x^{h-1} \eta
$$

is injective in $L^{2}(\mathbb{R})$, for every $\eta \neq 0$. As before, the change of variable $x \rightarrow|\eta|^{\frac{1}{h+1}} x$ leads to the $L^{2}(\mathbb{R})$-injectivity of the anharmonic oscillators $P_{ \pm \mu}=D_{x}^{2}+a x^{2 h} \pm$ $\mu x^{h-1}$, which are actually unitarily equivalent, via the antipodal map $x \rightarrow-x$.

Summing up, we have proved that

$$
P_{\mu}=-\partial_{x}^{2}+a x^{2 h}+\mu x^{h-1}
$$

is injective in $L^{2}(\mathbb{R})$ if and only if

$$
\begin{aligned}
& \text { - } \quad \frac{\mu-\sqrt{a} h}{2 \sqrt{a}} \neq j(h+1)+1 / 2, \quad \forall j \in \mathbb{Z}, \quad \text { when } h \text { is even; } \\
& \text { - } \quad-\frac{\mu+\sqrt{a} h}{2 \sqrt{a}} \neq\left\{\begin{array}{l}
j(h+1), \\
j(h+1)+1,
\end{array} \quad \forall j \in \mathbb{N}, \quad \text { when } h\right. \text { is odd. }
\end{aligned}
$$

Thus, by a straightforward computation, it is seen that $0 \in \operatorname{Spec}\left(P_{\mu}\right)=\left\{\lambda_{j}(\mu) \mid j \in\right.$ $\mathbb{N}\}$ if and only if, for some $j \in \mathbb{N}$, one has

(C1) $\mu=\sqrt{a}(h+1)(2 j+1)$ or $\mu=-\sqrt{a}(h+1)(2 j+1)$, if $h$ is even;

(C2) $\mu=(-1)^{j} \sqrt{a}-\sqrt{a}(h+1)(j+\theta(j))$, if $h$ is odd.

In what follows, we refer to these special values of $\mu$ as the critical values of $P_{\mu}$. If $\mu$ attains one of its critical values, then an eigenvalue $\lambda_{j}(\mu)$ in (1.3) must vanish, but we do not know which of them. In order to establish a precise relation between the critical values and the vanishing eigenvalues, we need the following lemma.

Lemma 2.1. Let $\bar{\mu} \in \mathbb{R}$ be a critical value of $P_{\mu}$, according to $(C 1)$ and $(C 2)$. Denote by $\lambda(\mu)$ the eigenvalue of $P_{\mu}$ such that $\lambda(\bar{\mu})=0$. Then:

(1) if $h$ is odd, $\lambda(\mu)$ is a strictly increasing function of $\mu$;

(2) if $h$ is even, $\lambda(\mu)$ is strictly increasing (resp., decreasing) locally near $\bar{\mu}$, if $\bar{\mu}$ is negative (resp., positive).

Proof. In Appendix I it is proved, among other classical results, that the eigenfunctions and the eigenvalues of $P_{\mu}$ are smooth (analytic) functions of the parameter $\mu$. Let $\phi_{\mu}(x) \in L^{2}(\mathbb{R})$ be a real normalized eigenfunction corresponding to the eigenvalue $\lambda(\mu)$.

By differentiating, with respect to $\mu$, both sides of the trivial identity $P_{\mu} \phi_{\mu}=$ $\lambda(\mu) \phi_{\mu}$, we get

$$
x^{h-1} \phi_{\mu}+P_{\mu}\left(\partial_{\mu} \phi_{\mu}\right)=\lambda^{\prime}(\mu) \phi_{\mu}+\lambda(\mu) \partial_{\mu} \phi_{\mu},
$$

and taking the inner product with $\phi_{\mu}$ yields

$$
\lambda^{\prime}(\mu)=\left\langle x^{h-1} \phi_{\mu}, \phi_{\mu}\right\rangle,
$$

being $\left\langle P_{\mu}\left(\partial_{\mu} \phi_{\mu}\right), \phi_{\mu}\right\rangle=\left\langle\partial_{\mu} \phi_{\mu}, P_{\mu}\left(\phi_{\mu}\right)\right\rangle=\lambda(\mu)\left\langle\partial_{\mu} \phi_{\mu}, \phi_{\mu}\right\rangle$ (this point is also discussed in Appendix I in the framework of the Rayleigh-Schrödinger series; see (6.6)).

If $h$ is odd, then one has that $\left\langle x^{h-1} \phi_{\mu}, \phi_{\mu}\right\rangle=\left\|x^{\frac{h-1}{2}} \phi_{\mu}\right\|_{L^{2}(\mathbb{R})}^{2}>0$, which concludes the proof. Finally, if $h$ is even, we observe that

$$
0=\left\langle P_{\bar{\mu}} \phi_{\bar{\mu}}, \phi_{\bar{\mu}}\right\rangle=\left\|\partial_{x} \phi_{\bar{\mu}}\right\|_{L^{2}(\mathbb{R})}^{2}+a\left\|x^{h} \phi_{\bar{\mu}}\right\|_{L^{2}(\mathbb{R})}^{2}+\bar{\mu}\left\langle x^{h-1} \phi_{\bar{\mu}}, \phi_{\bar{\mu}}\right\rangle,
$$


whence one immediately gets

$$
\left\langle x^{h-1} \phi_{\bar{\mu}}, \phi_{\bar{\mu}}\right\rangle=-\frac{\left\|\partial_{x} \phi_{\bar{\mu}}\right\|_{L^{2}(\mathbb{R})}^{2}+a\left\|x^{h} \phi_{\bar{\mu}}\right\|_{L^{2}(\mathbb{R})}^{2}}{\bar{\mu}} .
$$

Therefore, due to (2.3),$\lambda^{\prime}(\bar{\mu})$ has the same sign of $-\bar{\mu}$ and this concludes the proof of (2.4).

In order to prove Theorem 1.1 we proceed by induction on $j$. Firstly, we assume that $h$ is an even integer; we shall see that similar arguments apply to the odd case.

In view of the parity (1.8), we can limit ourselves to studying the sign of the eigenvalues $\lambda_{j}(\mu)$ for $\mu \geq 0$. Let us start off by proving (1.5) for $\lambda_{0}(\mu)$.

We observe that $P_{0}=-\partial_{x}^{2}+a x^{2 h}$ is a positive $L^{2}(\mathbb{R})$-operator, due to the trivial identity $\left\langle P_{0} u, u\right\rangle=\left\|\partial_{x} u\right\|_{L^{2}(\mathbb{R})}^{2}+a\left\|x^{h} u\right\|_{L^{2}(\mathbb{R})}^{2}>0$ (whenever $0 \neq u \in B_{h}$ ). As a consequence, we have that $\lambda_{j}(0)>0$ for every $j \in \mathbb{N}$.

Furthermore, $\lambda_{0}(\mu)$ is the lowest eigenvalue of $P_{\mu}$ and is a continuous function of $\mu$; thus, in view of $(C 1)$ above, we get

$$
\lambda_{0}(\mu)>0 \quad \text { if } \quad 0 \leq \mu<\sqrt{a}(h+1) \quad \text { and } \quad \lambda_{0}(\mu)=0 \quad \text { if } \quad \mu=\sqrt{a}(h+1) .
$$

In view of Lemma 2.1 there exists a positive constant $\epsilon$ such that

$$
\lambda_{0}(\mu)<0 \text { if } \sqrt{a}(h+1)<\mu<\sqrt{a}(h+1)+\epsilon \text {; }
$$

by taking into account the critical values $(C 1)$ and the continuity of $\lambda_{0}(\mu)$, we get

$$
\lambda_{0}(\mu)<0 \quad \text { if } \sqrt{a}(h+1)<\mu<3 \sqrt{a}(h+1) .
$$

By continuity we have $\lambda_{0}(3 \sqrt{a}(h+1)) \leq 0$, but if $\lambda_{0}(3 \sqrt{a}(h+1))=0$, then from Lemma 2.1 it would follow that $\lambda_{0}(\mu)>0$ for $3 \sqrt{a}(h+1)-\epsilon<\mu<3 \sqrt{a}(h+1)$, which is in contradiction with (2.5).

As a consequence, we have $\lambda_{0}(3 \sqrt{a}(h+1))<0$ and, in view of $(C 1), \lambda_{0}(\mu)$ never vanishes in the interval $3 \sqrt{a}(h+1)<\mu<5 \sqrt{a}(h+1)$; thus, the continuity of $\lambda_{0}$ yields

$$
\lambda_{0}(\mu)<0 \quad \text { if } \quad 3 \sqrt{a}(h+1)<\mu<5 \sqrt{a}(h+1) .
$$

An iteration of this procedure proves (1.5) for $j=0$.

Suppose now that (1.5) is true for every $j<k$. In order to get (1.5) for $j=k$ and $\mu \geq 0$, observe that $\lambda_{k}(\mu)>\lambda_{k-1}(\mu) \geq 0$ for $0 \leq \mu \leq \sqrt{a}(h+1)(2(k-1)+1)$ due to (1.3) and to the inductive hypothesis. By virtue of $(C 1), \lambda_{k}(\mu)$ never vanishes in the interval $\sqrt{a}(h+1)(2(k-1)+1)<\mu<\sqrt{a}(h+1)(2 k+1)$, and by continuity it follows that

$\lambda_{k}(\mu)>0$ whenever $0 \leq \mu<\sqrt{a}(h+1)(2 k+1)$, and $\lambda_{k}(\sqrt{a}(h+1)(2 k+1)) \geq 0$.

If $\lambda_{k}(\sqrt{a}(h+1)(2 k+1))>0$, by the inductive hypothesis we would have $\lambda_{j}(\sqrt{a}(h+1)(2 k+1))<0$ for any $j<k$ and, by (1.3), $\lambda_{j}(\sqrt{a}(h+1)(2 k+1))>0$ for any $j \geq k$; thus it would follow that $\lambda_{j}(\sqrt{a}(h+1)(2 k+1)) \neq 0$ for every $j \in \mathbb{N}$, which is impossible in view of $(C 1)$. Hence we get $\lambda_{k}(\sqrt{a}(h+1)(2 k+1))=0$.

In order to show that $\lambda_{k}(\mu)<0$ if $\mu>\sqrt{a}(h+1)(2 k+1)$, we can argue as for the eigenvalue $\lambda_{0}(\mu)$, so that Theorem 1.1 is proved for any even integer $h$.

For what concerns the odd case, the proof proceeds very closely to the even one and, again, is strongly based on the continuity of the eigenvalues $\lambda_{j}$. Since 
$P_{0}=-\partial_{x}^{2}+a x^{2 h}$ is an $L^{2}(\mathbb{R})$-positive operator, we have $\lambda_{0}(0)>0$ and, by virtue of $(C 2)$, one gets

$$
\lambda_{0}(\mu)>0 \quad \text { if } \quad \mu>\sqrt{a}-\sqrt{a}(h+1)=-\sqrt{a} h \quad \text { and } \quad \lambda_{0}(\mu)=0 \quad \text { if } \quad \mu=-\sqrt{a} h .
$$

Moreover, due to Lemma 2.1, $\lambda_{0}(\mu)$ is a strictly increasing function so that $\lambda_{0}(\mu)<$ 0 whenever $\mu<-\sqrt{a} h$. Finally, as in the even case, an inductive argument completes the proof of (1.6) for $j>0$.

It remains to discuss the $L^{2}(\mathbb{R})$-non-negativity (positivity) of $P_{\mu}$. Note that, due to (1.3), this property is equivalent to the non-negativity (positivity) of its lowest eigenvalue $\lambda_{0}(\mu)$; hence, (1.7) readily follows from (1.5) and (1.6) for $j=0$.

We are left with the proof of Corollary 1.2

Let us begin with the even case. Since $\lambda_{j}(\mu)$ is an analytic function that vanishes in $\mu_{j}^{+}=\sqrt{a}(h+1)(2 j+1)$, we have

$$
\lambda_{j}(\mu)=\lambda_{j}(\mu)-\lambda_{j}\left(\mu_{j}^{+}\right)=\int_{0}^{1} \frac{d}{d t} \lambda_{j}\left(t \mu+(1-t) \mu_{j}^{+}\right) d t=\psi(\mu)\left(\mu-\mu_{j}^{+}\right),
$$

where $\psi(\mu):=\int_{0}^{1} \lambda_{j}^{\prime}\left(t \mu+(1-t) \mu_{j}\right) d t$ is an analytic function of $\mu$. Again from Theorem 1.1 and from (2.6) it follows that $\psi(\mu)$ vanishes at $\mu_{j}^{-}=-\sqrt{a}(h+1)(2 j+1)$. By arguing as before, we obtain $\psi(\mu)=\tilde{\lambda}_{j}(\mu)\left(\mu-\mu_{j}^{-}\right)$, for a suitable analytic function $\tilde{\lambda}_{j}(\mu)$, so that

$$
\lambda_{j}(\mu)=\tilde{\lambda}_{j}(\mu)\left(\mu-\mu_{j}^{-}\right)\left(\mu-\mu_{j}^{+}\right)=\tilde{\lambda}_{j}(\mu)\left(\mu^{2}-a(h+1)^{2}(2 j+1)^{2}\right) .
$$

By Theorem 1.1 it is easily seen that $\tilde{\lambda}_{j}(\mu)<0$ if $\mu \neq \mu_{j}^{ \pm}$, and we are thus left to evaluate $\tilde{\lambda}_{j}\left(\mu_{j}^{ \pm}\right)$. To this end, from the identity (2.3) we obtain

$$
\lambda_{j}^{\prime}\left(\mu_{j}^{ \pm}\right)=\left\langle x^{h-1} \phi_{\mu_{j}^{ \pm}}, \phi_{\mu_{j}^{ \pm}}\right\rangle=-\frac{\left\|\partial_{x} \phi_{\mu_{j}^{ \pm}}\right\|_{L^{2}(\mathbb{R})}^{2}+a\left\|x^{h} \phi_{\mu_{j}^{ \pm}}\right\|_{L^{2}(\mathbb{R})}^{2}}{\mu_{j}^{ \pm}},
$$

where $\phi_{\mu_{j}^{ \pm}}$is a normalized eigenfunction in $\operatorname{Ker}\left(P_{\mu_{j}^{ \pm}}-\lambda_{j}\left(\mu_{j}^{ \pm}\right) I\right)$. On the other hand, from (2.7) it follows that

$$
\lambda_{j}^{\prime}\left(\mu_{j}^{ \pm}\right)=2 \mu_{j}^{ \pm} \tilde{\lambda}_{j}\left(\mu_{j}^{ \pm}\right)
$$

and summing up yields

$$
\tilde{\lambda}_{j}\left(\mu_{j}^{ \pm}\right)=-\frac{\left\|\partial_{x} \phi_{\mu_{j}^{ \pm}}\right\|_{L^{2}(\mathbb{R})}^{2}+a\left\|x^{h} \phi_{\mu_{j}^{ \pm}}\right\|_{L^{2}(\mathbb{R})}^{2}}{2\left(\mu_{j}^{ \pm}\right)^{2}}<0 .
$$

Finally, the parity of $\tilde{\lambda}_{j}(\mu)$ is a trivial consequence of (1.8).

As for the odd case, arguing as above gives

$$
\lambda_{j}(\mu)=\tilde{\lambda}_{j}(\mu)\left(\mu_{j}-\mu\right) \quad \text { and } \quad \tilde{\lambda}_{j}\left(\mu_{j}\right)=-\lambda_{j}^{\prime}\left(\mu_{j}\right)=-\left\langle x^{h-1} \phi_{\mu_{j}}, \phi_{\mu_{j}}\right\rangle,
$$

where $\tilde{\lambda}_{j}(\mu)$ is an analytic function of $\mu$ and $\mu_{j}=(-1)^{j} \sqrt{a}-\sqrt{a}(h+1)(j+\theta(j))$. Since $h$ is odd, the term $-\left\langle x^{h-1} \phi_{\mu_{j}}, \phi_{\mu_{j}}\right\rangle=-\int x^{h-1}\left|\phi_{\mu_{j}}(x)\right|^{2} d x$ is strictly negative, so that the proof is complete.

Remark 2.2. We point out that, unlike the even case, if $h$ is odd, the normalized eigenfunctions $\phi_{\mu_{j}}$ in the kernel of $P_{\mu_{j}}$ are completely known (we address the reader to Proposition 2.1 in [14] for the precise description); thus, the value $\tilde{\lambda}_{j}\left(\mu_{j}\right)=$ $-\left\langle x^{h-1} \phi_{\mu_{j}}, \phi_{\mu_{j}}\right\rangle$ can be "explicitly" computed. Note that the operator (2.2) in [14] 
reduces to the anharmonic oscillator $P_{\mu}$ only for $a=1$; however, by performing the change of variable $y=a^{-\frac{1}{h+1}} x$ in $(2.2)$, we easily get $P_{\mu}$ in the general case $a \in \mathbb{R}_{+}$.

\section{Behaviour of the eigenvalues of $P_{\mu}$}

Our aim is to analyse the qualitative behaviour of the eigenvalues $\lambda_{j}(\mu)$ of $P_{\mu}$ as $\mu$ varies in $\mathbb{R}$. In particular, we are interested in finding out the extrema and the points of local minimum or maximum of the $\lambda_{j}(\mu)$.

In 18, and 19, this analysis is carried out for the lowest eigenvalue of the anharmonic oscillator $-\partial_{x}^{2}+\left(x^{h} / h-\alpha\right)^{2}, \alpha \in \mathbb{R}$. However, as we shall see in this section, the behaviour of our $\lambda_{0}(\mu)$ is here rather different, despite the fact that the potential $x^{2 h} / h^{2}-2(\alpha / h) x^{h}+\alpha^{2}$ and $x^{2 h} / h^{2}-2(\alpha / h) x^{h-1}$ seem similar.

We start off by studying the asymptotic behaviour of the eigenvalues $\lambda_{j}(\mu)$ of $P_{\mu}$ as $\mu \longrightarrow \infty$.

Proposition 3.1. If $h$ is even, we have that, for any $j \in \mathbb{N}$,

$$
\lambda_{j}(\mu)=K_{\min }|\mu|^{\frac{2 h}{h+1}}+e_{j}|\mu|^{\frac{h-1}{h+1}}+O\left(|\mu|^{-\frac{2}{h+1}}\right), \quad \mu \longrightarrow \pm \infty,
$$

where

$$
\begin{aligned}
K_{\min } & :=\min _{x \in \mathbb{R}}\left\{a x^{2 h}-x^{h-1}\right\}=-\frac{h+1}{2 h}\left(\frac{h-1}{2 h a}\right)^{\frac{h-1}{h+1}}<0, \\
e_{j} & :=\sqrt{\frac{h+1}{2}}(2 h a)^{-\frac{h-3}{2(h+1)}}(h-1)^{\frac{h-1}{h+1}}(2 j+1) .
\end{aligned}
$$

Assume that $h$ is odd. In this case the potential ax $x^{2 h}-x^{h-1}$ has symmetric double wells so that the consecutive eigenvalues $\lambda_{2 j}(\mu)$ and $\lambda_{2 j+1}(\mu)$ have the same asymptotic behaviour as $\mu \longrightarrow-\infty$ :

$$
\begin{aligned}
\lambda_{2 j}(\mu) & =K_{\min }|\mu|^{\frac{2 h}{h+1}}+e_{j}|\mu|^{\frac{h-1}{h+1}}+O\left(|\mu|^{-\frac{2}{h+1}}\right), \\
\lambda_{2 j+1}(\mu) & =K_{\min }|\mu|^{\frac{2 h}{h+1}}+e_{j}|\mu|^{\frac{h-1}{h+1}}+O\left(|\mu|^{-\frac{2}{h+1}}\right) .
\end{aligned}
$$

As for $\mu \longrightarrow+\infty$, consider the "reduced" anharmonic oscillator $Q_{0}=D_{x}^{2}+x^{h-1}$ (here $h-1$ is even). Its spectrum is made of the simple eigenvalues $\nu_{j} \in \mathbb{R}_{+}$such that

$$
0<\nu_{0}<\nu_{1}<\cdots<\nu_{j}<\nu_{j+1}<\cdots, \quad j \in \mathbb{N} .
$$

Denote by $\psi_{j}(x) \in B_{\frac{h-1}{2}}($ see $(1.2))$ an $L^{2}(\mathbb{R})$-normalized eigenfunction of $Q_{0}$ corresponding to the eigenvalue $\nu_{j}$ (as a matter of fact, it turns out that $\psi_{j}(x) \in$ $\mathcal{S}(\mathbb{R}))$. Then we have that, for any $j \in \mathbb{N}$,

$$
\lambda_{j}(\mu)=\nu_{j} \mu^{\frac{2}{h+1}}+\left\langle x^{2 h} \psi_{j}, \psi_{j}\right\rangle \mu^{-\frac{2 h}{h+1}}+O\left(\mu^{-\frac{4 h+2}{h+1}}\right), \quad \mu \longrightarrow+\infty .
$$

Note that, if $h$ is odd, the eigenvalues $\lambda_{j}(\mu)$ behave very different according to whether $\mu \longrightarrow-\infty$ or $\mu \longrightarrow+\infty$. This fact is a direct consequence of the geometry of the potential $V_{\mu}(x)=a x^{2 h}+\mu x^{h-1}$ : if $\mu<0$, the absolute minimum of $V_{\mu}(x)$ is negative and non-degenerate, whereas, if $\mu>0$, its absolute minimum is zero and is degenerate (of order $h-1$ ).

Proof of Proposition 3.1. Let us first assume that $\mu<0$. If we perform the change of variable $x \longrightarrow|\mu|^{-\frac{1}{h+1}} x$, the anharmonic oscillator $P_{\mu}$ is turned into

$$
\widetilde{P}_{\mu}=|\mu|^{-\frac{2}{h+1}}\left(D_{x}^{2}+\mu^{2}\left(a x^{2 h}-x^{h-1}\right)\right),
$$


which has the same spectrum as $P_{\mu}$. An easy computation shows that

$$
K_{\min }:=\min _{x \in \mathbb{R}}\left\{a x^{2 h}-x^{h-1}\right\}=-\frac{h+1}{2 h}\left(\frac{h-1}{2 h a}\right)^{\frac{h-1}{h+1}}<0 .
$$

In order to deal with a non-negative potential $V(x)$, let us write

$$
\widetilde{P}_{\mu}=|\mu|^{-\frac{2}{h+1}}\left(D_{x}^{2}+\mu^{2}(\underbrace{a x^{2 h}-x^{h-1}-K_{\min }}_{=V_{-}(x)})\right)+K_{\min }|\mu|^{\frac{2 h}{h+1}},
$$

and note that the $V_{-}(x)$ attains its absolute minimum 0 at the unique point $x_{e}:=$ $\sqrt[h+1]{\frac{h-1}{2 h a}}$, if $h$ is even, and at the opposite points $x_{o}^{ \pm}:= \pm \sqrt[h+1]{\frac{h-1}{2 h a}}$, if $h$ is odd. Moreover, in each case, the origin is a non-degenerate minimum for

$$
V_{-}^{\prime \prime}(\bar{x})=(2 h a)^{-\frac{h-3}{h+1}}(h-1)^{\frac{2 h-2}{h+1}}(h+1)>0,
$$

where $\bar{x}=x_{e}$ or $\pm x_{o}$, according to the parity of $h$. As pointed out in 34] (see also [17]), the Schrödinger operator $D_{x}^{2}+\mu^{2}\left(a x^{2 h}-x^{h-1}-K_{\min }\right)$ looks like the harmonic oscillator $|\mu|\left(D_{x}^{2}+\frac{1}{2} V_{-}^{\prime \prime}(\bar{x}) x^{2}\right)$, as $\mu \longrightarrow-\infty$; thus the eigenvalues of the former are asymptotically equivalent to the ones of the latter. Precisely, denote by $\tilde{\lambda}_{0}(\mu)<\widetilde{\lambda}_{1}(\mu)<\cdots$ the eigenvalues of $D_{x}^{2}+\mu^{2}\left(a x^{2 h}-x^{h-1}-K_{\min }\right)$ and by $e_{j}$ those of $\left(D_{x}^{2}+\frac{1}{2} V_{-}^{\prime \prime}(\bar{x}) x^{2}\right)$, i.e.,

$$
e_{j}=\sqrt{\frac{1}{2} V_{-}^{\prime \prime}(\bar{x})}(2 j+1)=\sqrt{\frac{h+1}{2}}(2 h a)^{-\frac{h-3}{2(h+1)}}(h-1)^{\frac{h-1}{h+1}}(2 j+1) .
$$

If $h$ is even, the potential $V_{-}(x)$ has a single well; thus from Theorem 4.1 34 it follows that

$$
\widetilde{\lambda}_{j}(\mu)=e_{j}|\mu|+O(1), \quad \mu \longrightarrow-\infty .
$$

In view of (3.5), we have the identity

$$
\lambda_{j}(\mu)=K_{\min }|\mu|^{\frac{2 h}{h+1}}+|\mu|^{-\frac{2}{h+1}} \widetilde{\lambda}_{j}(\mu),
$$

whence, due to (1.8), (3.1) immediately follows.

On the other hand, if $h$ is odd, $V_{-}(x)$ has double symmetric wells and, as a consequence, the eigenvalues tend to group in pairs; indeed, by Theorem 5.1 in 34 (see "Errata" in [34]) the eigenvalues $\widetilde{\lambda}_{2 j}(\mu)$ and $\widetilde{\lambda}_{2 j+1}(\mu)$ have the same asymptotic growth $e_{j}|\mu|+O(1)$ as $\mu \longrightarrow-\infty$, so that, by (3.6), (3.2) is proved.

In order to study the behaviour of the $\lambda_{j}(\mu)$ when $\mu \longrightarrow+\infty$ and $h$ is odd, we apply to $P_{\mu}$ the change of variable $x \longrightarrow \mu^{\frac{1}{h+1}} x$ and we get the unitarily equivalent operator

$$
\widetilde{P}_{\mu}=\mu^{\frac{2}{h+1}}\left(D_{x}^{2}+x^{h-1}+\frac{1}{\mu^{2}} a x^{2 h}\right)=\mu^{\frac{2}{h+1}}\left(H_{0}+\epsilon V\right),
$$

where we have set $H_{0}=D_{x}^{2}+x^{h-1}, V=a x^{2 h}, \epsilon=1 / \mu^{2}$. We are so left to study the asymptotic behaviour of the eigenvalues $\left\{\nu_{j}(\epsilon)\right\}_{j \in \mathbb{N}}$ of $H_{0}+\epsilon V$ as $\epsilon \rightarrow 0^{+}$. Observe that the $\nu_{j}:=\nu_{j}(0)$ are exactly the eigenvalues of $H_{0}$, which are supposed to be arranged as in (3.3).

The standard "regular" perturbation theory (see Appendix I) does not apply to $H_{0}+\epsilon V$, since $V$ is not a "small" perturbation of $H_{0}$. In fact, the natural domain $B_{\frac{h-1}{2}}$ of $H_{0}$ (see (1.2) ) immediately changes as soon as the perturbation $V$ is turned on, for $\mathcal{D}\left(H_{0}+\epsilon V\right)=B_{h} \varsubsetneqq B_{\frac{h-1}{2}}$, whenever $\epsilon>0$. However, as shown in Section III.1 and in Theorem A.II.2 of [33], upon denoting $V_{0}=x^{h-1}, V_{\text {pert }}=a x^{2 h}$, 
the asymptotic behaviour of the eigenvalue $\nu_{j}(\epsilon)$, as $\epsilon \rightarrow 0^{+}$, is described by the Rayleigh-Schrödinger series (6.4)

$$
\nu_{j}(\epsilon) \sim \nu_{j}(0)+\sum_{s=0}^{+\infty} \epsilon^{s+1}\left\langle V A_{s} \phi_{j}, \phi_{j}\right\rangle,
$$

where $\psi_{j}(x) \in B_{\frac{h-1}{2}}$ (see (1.2)) is an $L^{2}(\mathbb{R})$-normalized eigenfunction corresponding to the eigenvalue $\nu_{j}(0)=: \nu_{j}$. Therefore, from (6.5) it follows that

$$
\nu_{j}(\epsilon)=\nu_{j}+\epsilon\left\langle V \psi_{j}, \psi_{j}\right\rangle+O\left(\epsilon^{2}\right) .
$$

By taking into account (3.7), we finally conclude that

$$
\lambda_{j}(\mu)=\nu_{j} \mu^{\frac{2}{h+1}}+\left\langle x^{2 h} \psi_{j}, \psi_{j}\right\rangle \mu^{-\frac{2 h}{h+1}}+O\left(\mu^{-\frac{4 h+2}{h+1}}\right), \quad \mu \longrightarrow+\infty .
$$

As a straightforward consequence of Proposition 3.1 we see that, for any $j \in \mathbb{N}$,

$$
\begin{gathered}
\lim _{\mu \longrightarrow+\infty} \lambda_{j}(\mu)=-\infty \quad \text { if } h \text { is even, } \\
\lim _{\mu \longrightarrow-\infty} \lambda_{j}(\mu)=-\infty, \quad \lim _{\mu \longrightarrow+\infty} \lambda_{j}(\mu)=+\infty, \quad \text { if } h \text { is odd. }
\end{gathered}
$$

Thus, by virtue of (3.9) and (1) of Lemma 2.1, we have the following

Corollary 3.2. If $h$ is odd, then every eigenvalue $\lambda_{j}(\mu)$ does not admit any point of local minimum or maximum. Furthermore, one has that $\inf _{\mathbb{R}} \lambda_{j}=-\infty$ and $\sup _{\mathbb{R}} \lambda_{j}=+\infty$.

If $h$ is even, the picture is more complicated. Due to (3.8) we know that $\inf _{\mathbb{R}} \lambda_{j}=$ $-\infty$ and that there exists (at least) a $\mu_{\max }^{(j)} \in \mathbb{R}$ for which

$$
\sup _{\mathbb{R}} \lambda_{j}=\lambda_{j}\left(\mu_{\max }^{(j)}\right) .
$$

Moreover, if $\phi_{j, \mu}$ is a normalized eigenfunction associated with $\lambda_{j}(\mu)$, we trivially have

$$
\lambda_{j}(\mu)=\left\langle P_{\mu} \phi_{j, \mu}, \phi_{j, \mu}\right\rangle=\left\|\partial_{x} \phi_{j, \mu}\right\|_{L^{2}(\mathbb{R})}^{2}+a\left\|x^{h} \phi_{j, \mu}\right\|_{L^{2}(\mathbb{R})}^{2}+\mu\left\langle x^{h-1} \phi_{j, \mu}, \phi_{j, \mu}\right\rangle .
$$

By using (2.3) (see also (6.6) ) we obtain

$$
\lambda_{j}^{\prime}(\mu)=\left\langle x^{h-1} \phi_{j, \mu}, \phi_{j, \mu}\right\rangle=\frac{\lambda_{j}(\mu)-\left\|\partial_{x} \phi_{j, \mu}\right\|_{L^{2}(\mathbb{R})}^{2}-a\left\|x^{h} \phi_{j, \mu}\right\|_{L^{2}(\mathbb{R})}^{2}}{\mu} .
$$

In view of (1.5), it follows that the eigenvalue $\lambda_{j}(\mu)$ is strictly increasing if $\mu<$ $-\sqrt{a}(h+1)(2 j+1)$ and is strictly decreasing if $\mu>\sqrt{a}(h+1)(2 j+1)$. As a consequence, the only possible points of local minimum or maximum lie inside the interval $|\mu|<\sqrt{a}(h+1)(2 j+1)$ and hence $\left|\mu_{\max }^{(j)}\right|<\sqrt{a}(h+1)(2 j+1)$.

From (1.8) it follows that the first derivative of $\lambda_{j}$ is an odd function, so that $\lambda_{j}^{\prime}(0)=0$. Moreover, $\lambda_{j}(\mu)$ is a (non-constant) analytic function at $\mu=0$, hence $\mu=0$ must be a strict local minimum or maximum according to the sign of its $m$-th derivative $\lambda_{j}^{(m)}(0)$, where $m=\min \left\{n \in \mathbb{N} \mid \lambda_{j}^{(n)}(0) \neq 0\right\}$.

Unfortunately, we are not able to establish the precise nature of the origin, for a general eigenvalue $\lambda_{j}(\mu)$; furthermore, we do not know if there are other critical points inside the interval $|\mu|<\sqrt{a}(h+1)(2 j+1)$. 
However, if we limit ourselves to consider the ground state energy $\lambda_{0}(\mu)$ of $P_{\mu}$, we have the following result.

Lemma 3.3. Assume that $h$ is even. Then

(1) $\lambda_{0}(\mu)$ is a strictly concave function;

(2) its maximum is attained only at the origin; namely, $\mu_{\max }^{(0)}=0$ and $\sup _{\mathbb{R}} \lambda_{0}=$ $\lambda_{0}(0)$

(3) the origin is the only critical point of $\lambda_{0}(\mu)$ and is non-degenerate.

Proof. As noted above, the origin is a critical point of $\lambda_{0}(\mu)$; the proof is thus a straightforward consequence of (6.10).

\section{LOWER BOUNDS}

In this section we discuss the existence of an $L^{2}$-lower bound for the quadratic form associated with a (pseudo)differential operator (see (4.3) below with $m=2$ ). In order to introduce our problem, let us briefly recall some classical results about lower bounds.

Let $\Omega \subset \mathbb{R}^{n}$ be an open set and $Q=q(z, D) \in \mathrm{OP} S^{m}\left(\mathbb{R}^{n}\right)$ be a classical formally self-adjoint $\psi$ do with $q(z, \zeta) \sim \sum_{j>0} q_{m-j}(z, \zeta)$. For the sake of simplicity we assume that $m>0$.

It is well-known (Gårding Inequality) that $Q$ is positive elliptic (namely, $q_{m}(z, \zeta)$ $>0$ for $\left.(z, \zeta) \in T^{*} \Omega \backslash 0\right)$ if and only if, for every compact set $K \subset \Omega$, there exist two positive constants $c, C$ for which

$$
\langle Q u, u\rangle \geq c\|u\|_{m / 2}^{2}-C\|u\|_{0}^{2}, \quad \forall u \in C_{0}^{\infty}(K),
$$

where $\|u\|_{s}$ denotes the usual $H^{s}$-Sobolev norm of $u$.

If the principal symbol $q_{m}(x, \zeta)$ vanishes, i.e., $q_{m}(z, \zeta) \geq 0$, it was proved by Hörmander 20] that $Q$ satisfies, in general, only a weaker inequality, the so-called Sharp Gårding Inequality:

$$
\langle Q u, u\rangle \geq-C\|u\|_{\frac{m-1}{2}}^{2}, \quad \forall u \in C_{0}^{\infty}(K) .
$$

We point out that (4.1) is stable under perturbation of $Q$ with operators of order $\leq(m-1)$. If we are interested in improving this inequality, we are forced to consider the impact of the lower terms $q_{m-j}(z, \zeta)$.

Note that, due to the non-negativity, $q_{m}$ vanishes (at least) to the second order on its characteristic set $\Sigma=\left\{(z, \zeta) \in T^{*} \Omega \backslash 0 \mid q_{m}(z, \zeta)=0\right\}$. We say that $Q$ is transversally elliptic, if $q_{m}$ vanishes exactly to the second order on $\Sigma$; namely,

$$
\left|q_{m}(z, \zeta)\right| \approx|\zeta|^{m} \operatorname{dist}_{\Sigma}(z, \zeta)^{2}
$$

where $\operatorname{dist}_{\Sigma}(z, \zeta)$ denotes the distance of $(z, \zeta /|\zeta|)$ to $\Sigma$. In [21] Hörmander improved the Sharp Gårding Inequality in the case of transversally elliptic operators $Q$, for which $\Sigma$ is a smooth submanifold of $T^{*} \Omega \backslash 0$ and the symplectic 2 -form $\sigma=\sum_{j=1}^{n} d \xi_{j} \wedge d x_{j}$ has constant rank on the connected components of $\Sigma$ (see Chapter III in [21]). In this set-up, he proved that

$$
\langle Q u, u\rangle \geq-C_{K}\|u\|_{m / 2-1}^{2}, \quad \forall u \in C_{0}^{\infty}(K),
$$

holds for any compact set $K \subset \Omega$, if and only if

$$
\varrho \in \Sigma \Longrightarrow q_{m-1}^{s}(\varrho)+\operatorname{Tr}^{+} F_{q_{m}}(\varrho) \geq 0,
$$


where

$$
q_{m-1}^{s}(\varrho):=q_{m-1}(\varrho)+\frac{i}{2} \sum_{j=1}^{n} \frac{\partial^{2} q_{m}}{\partial z_{j} \partial \zeta_{j}}(\varrho)
$$

is the subprincipal symbol of $Q$, and $\operatorname{Tr}^{+} F_{q_{m}}(\varrho)$ is the positive trace of the fundamental matrix associated with $Q$ at $\varrho \in \Sigma$, defined by

$$
\sigma\left(v, F_{q_{m}}(\varrho) w\right)=\frac{1}{2}\left\langle\operatorname{Hess} q_{m}(\varrho) v, w\right\rangle, \quad \forall v, w \in T_{\varrho} T^{*} \Omega .
$$

Now we want to show that hypothesis (4.4) is not, in general, enough to ensure (4.3) if one removes the transversal ellipticity condition (4.2). In [31 this fact is studied in a quite general setting and requires highly non-trivial technical machinery; our aim here is to discuss a simple example by using only "basic tools".

Let $h \in \mathbb{N}, a \in \mathbb{R}_{+}, b \in \mathbb{R}$ and consider the following Grushin-type operator in $\mathbb{R}_{(x, y)}^{2}$ :

$$
Q=D_{x}^{2}+a x^{2 h} D_{y}^{2}+b x^{h-1} D_{y}, \quad D=\frac{1}{\sqrt{-1}} \partial .
$$

Its principal symbol $q_{2}(x, y, \xi, \eta)=\xi^{2}+a x^{2 h} \eta^{2} \geq 0$ vanishes on the symplectic manifold $\Sigma=\{(x, y, \xi, \eta) \mid x=0=\xi\}$, and it is easily seen that, conically near $\Sigma$, $\operatorname{dist}_{\Sigma}(x, y, \xi, \eta) \approx\left(|x|+\frac{|\xi|}{|\eta|}\right)$. Thus,

$$
q_{2}(x, y, \xi, \eta)=\xi^{2}+a x^{2 h} \eta^{2} \approx \eta^{2}\left(|x|^{h}+\frac{|\xi|}{|\eta|}\right)^{2}
$$

turns out to be transversally elliptic (see (4.2) ) only if $h=1$. In this case, condition (4.4) reads as $|\mu| \leq \sqrt{a}$ and yields (4.3). On the other hand, if $h>1$, (4.4) is automatically verified for $q_{1}^{s} \equiv 0, \operatorname{Tr}^{+} F_{q_{2}} \equiv 0$ on $\Sigma$, but, as we shall see, (4.3) is, in general, false.

It is worth noting that the choice of the coefficient $x^{h-1}$ in the first order term of (4.5) is forced; in fact, as we shall see later on, the Hörmander inequality (4.3) does not hold for any power $x^{\gamma}$ with $0 \leq \gamma<h-1$ (unless $b=0$ ).

Assume that the operator (4.5) satisfies (4.3) for any compact neighborhood $K \subset \mathbb{R}^{2}$ of the origin. Let us choose $0 \neq \eta \in \mathbb{R}, 1 \leq t \in \mathbb{R}$ and $\phi(x), v(y) \in C_{0}^{\infty}(\mathbb{R})$ such that $\phi(t x) v(y) \in C_{0}^{\infty}(K)$ for $t$ large enough and $\int_{\mathbb{R}}|v(y)|^{2} d y=1$. We have, for any $1<t \in \mathbb{R}$, that

$$
\begin{gathered}
Q\left(e^{i t^{h+1} \eta y} \phi(t x) v(y)\right)=e^{i t^{h+1} \eta y}\left(\left(t^{2} D_{x}^{2}+a \eta^{2} t^{2 h+2} x^{2 h}+b \eta t^{h+1} x^{h-1}\right)(\phi)(t x) v(y)\right. \\
\left.+2 t^{h+1} a \eta x^{2 h} \phi(t x) D_{y} v+a x^{2 h} \phi(t x) D_{y}^{2} v+b x^{h-1} \phi(t x) D_{y} v\right) .
\end{gathered}
$$

Hence, by applying the change of variable $z=t x$, we get

$$
\begin{aligned}
& \left\langle Q\left(e^{i t^{h+1} \eta y} \phi(t x) v(y)\right), e^{i t^{h+1} \eta y} \phi(t x) v(y)\right\rangle \\
& \quad=t\left\langle\left(D_{z}^{2}+a \eta^{2} z^{2 h}+b \eta z^{h-1}\right) \phi(z) v(y), \phi(z) v(y)\right\rangle+o(t) \\
& \quad=t\left\langle\left(D_{z}^{2}+a \eta^{2} z^{2 h}+b \eta z^{h-1}\right) \phi(z), \phi(z)\right\rangle_{L^{2}(\mathbb{R})}+o(t),
\end{aligned}
$$

and

$$
\left\|e^{i t^{h+1} \eta y} \phi(t x) v(y)\right\|_{0}^{2}=t^{-1}\|\phi v\|_{0}^{2}=t^{-1}\|\phi\|_{L^{2}(\mathbb{R})}^{2} .
$$

Inserting the above relations in (4.3), dividing by $t$ and letting $t \longrightarrow+\infty$ lead to

$$
\left\langle\left(D_{z}^{2}+a \eta^{2} z^{2 h}+b \eta z^{h-1}\right) \phi(z), \phi(z)\right\rangle_{L^{2}(\mathbb{R})} \geq 0, \quad \forall \phi \in C_{0}^{\infty}(\mathbb{R}) .
$$


By choosing $\eta= \pm 1$ we deduce that $P_{ \pm b}=D_{z}^{2}+a z^{2 h} \pm b z^{h-1}$ is an $L^{2}(\mathbb{R})$-nonnegative operator; by virtue of the last part of Theorem 1.1 this is equivalent to the following explicit condition:

$$
|b| \leq h \sqrt{a} \text { if } h \text { is odd and }|b| \leq(h+1) \sqrt{a} \text { if } h \text { is even. }
$$

So far we have proved that (4.6) is a necessary condition in order for the operator $Q$ in (4.5) to satisfy the Hörmander inequality (4.3). As a consequence, if $h>1$ and $|b|$ is chosen greater enough, $Q$ violates (4.3) although hypothesis (4.4) is trivially verified. Therefore, in the Hörmander theorem, the transversal ellipticity condition cannot be a priori omitted.

If we replace the coefficient $x^{h-1}$ in the operator $Q$ by any power $x^{\gamma}$ with $0 \leq$ $\gamma<h-1$ and we apply the same localization argument used above, we obtain that

$$
b \eta t^{h-\gamma}\left\langle z^{\gamma} \phi(z) v(y), \phi(z) v(y)\right\rangle+o\left(t^{h-\gamma}\right) \geq t^{-1}\|\phi v\|_{0}^{2},
$$

whence, by setting again $\eta= \pm 1$,

$$
\pm b\left\langle z^{\gamma} \phi(z), \phi(z)\right\rangle_{L^{2}(\mathbb{R})} \geq 0, \quad \forall \phi \in C_{0}^{\infty}(\mathbb{R}),
$$

which gives $b=0$. It is exactly this argument that suggests to us the choice of the first order term in (4.5).

We are now going to show that the transversal ellipticity condition, although important, is not actually necessary to have (4.3). To this aim, it suffices to prove that (4.6) is indeed sufficient to have (4.3).

Observe that, by using the Parserval formula with respect to the $y$-variable, one has

$$
\langle Q u, u\rangle=(2 \pi)^{-1}\left\langle\left(D_{x}^{2}+a \eta^{2} x^{2 h}+b \eta x^{h-1}\right) \widehat{u}(x, \eta), \widehat{u}(x, \eta)\right\rangle,
$$

where $\widehat{u}(x, \eta)$ denotes the Fourier transform of $u \in C_{0}^{\infty}(K)$ with respect to the $y$-variable.

Performing the change of variable $z=|\eta|^{1 /(h+1)} x$ yields

$$
\begin{aligned}
\langle Q u, u\rangle= & (2 \pi)^{-1} \int|\eta|^{1 /(h+1)} \int\left(D_{z}^{2}+a z^{2 h}+\operatorname{sgn}(\eta) b z^{h-1}\right) \widehat{u}\left(|\eta|^{-1 /(h+1)} z, \eta\right) \\
& \cdot \bar{u}\left(|\eta|^{-1 /(h+1)} z, \eta\right) d z d \eta \\
= & (2 \pi)^{-1} \int|\eta|^{1 /(h+1)}\left\langle P_{\mu} \widehat{u}\left(|\eta|^{-1 /(h+1)} z, \eta\right), \widehat{u}\left(|\eta|^{-1 /(h+1)} z, \eta\right)\right\rangle_{L^{2}\left(\mathbb{R}_{z}\right)} d \eta
\end{aligned}
$$

where $\operatorname{sgn}(\eta)$ denotes the sign function of $\eta$ and $P_{\mu}$ is the operator (1.1) in the $z$-variable, with $\mu=\operatorname{sgn}(\eta) b$. Since $\lambda_{0}(\mu)$ is the lowest eigenvalue of the spectrum of $P_{\mu}$, one has $P_{\mu} \geq \lambda_{0}(\mu)$, whence it readily follows that

$$
\langle Q u, u\rangle \geq(2 \pi)^{-1} \min \left\{\lambda_{0}(b), \lambda_{0}(-b)\right\} \int|\eta|^{1 /(h+1)} \int\left|\widehat{u}\left(|\eta|^{-1 /(h+1)} z, \eta\right)\right|^{2} d z d \eta .
$$

By going back to the $x$-variable and by again applying the Parseval formula we obtain

$$
\langle Q u, u\rangle \geq(2 \pi)^{-2} \min \left\{\lambda_{0}(b), \lambda_{0}(-b)\right\} \iint|\eta|^{2 /(h+1)}|\widehat{u}(\xi, \eta)|^{2} d \xi d \eta .
$$

Therefore, the Hörmander inequality (4.3) easily follows if $\min \left\{\lambda_{0}(b), \lambda_{0}(-b)\right\} \geq 0$; in view of Theorem 1.1, this is exactly equivalent to (4.6). 
Note that, conically near $\Sigma$, we have $|\xi| \lesssim|\eta|$ so that $|\eta| \approx|(\xi, \eta)|$, whereas, outside $\Sigma, Q$ is microlocally elliptic; hence, from (4.7) we get, for a suitable positive constant $C$,

$$
\langle Q u, u\rangle \geq C \min \left\{\lambda_{0}(b), \lambda_{0}(-b)\right\} \iint|(\xi, \eta)|^{2 /(h+1)}|\widehat{u}(\xi, \eta)|^{2} d \xi d \eta,
$$

and if $\min \left\{\lambda_{0}(b), \lambda_{0}(-b)\right\}>0$ (i.e., the inequalities in (4.6) hold strictly) the following stronger estimate holds:

$$
\langle Q u, u\rangle \geq c\|u\|_{1 /(h+1)}^{2}-C^{\prime}\|u\|_{0}^{2}, \quad \forall u \in C_{0}^{\infty}(K),
$$

where $c, C^{\prime}$ are positive constants depending on $K$.

\section{Hypoellipticity}

In this section we apply Theorem 1.1 in some questions arising from the study of the hypoellipticity of non-transversally elliptic operators.

Precisely, here we study the $C^{\infty}$-hypoellipticity of the Grushin-type operator

$$
G=L^{*} L+\alpha(x, y)\left[L^{*}, L\right]=D_{x}^{2}+x^{2 h} D_{y}^{2}-(1+2 \alpha(x, y)) h x^{h-1} D_{y},
$$

where $L$ is the Mizohata operator $D_{x}-i x^{h} D_{y}$ and $\alpha(x, y) \in C^{\infty}\left(\mathbb{R}^{2}, \mathbb{R}\right)$.

From now on, for the sake of brevity, we assume that $h>1$ is an odd integer, so that $G$ is not transversally elliptic.

If $\alpha(x, y) \equiv \alpha$ is constant, the $C^{\infty}$-hypoellipticity of $G$ is completely studied by Gilioli-Treves in 11] and in [12, where it is proved that $G$ is $C^{\infty}$-hypoelliptic if and only if

$$
1+2 \alpha \neq \pm\left((-1)^{j+1} / h+(1+1 / h)(j+\theta(j))\right), \quad \forall j \in \mathbb{N},
$$

where, as usual, $\theta(j):=1$ if $j$ is even and $\theta(j):=0$ if $j$ is odd.

Precisely, in this case $G$ is hypoelliptic with loss of $\delta=2 h /(h+1)$ derivatives; namely,

$$
G u \in H_{l o c}^{s} \Longrightarrow u \in H_{l o c}^{2+s-\delta} .
$$

More recently, in Theorem 1 in [9] (see also the references therein) Cordaro and Hanges have shown that the assumptions (5.2) are necessary and sufficient also for the analytic hypoellipticity of $G$.

On the other hand, if $\alpha(x, y)$ is not constant, condition (5.2) is no longer necessary to guarantee the hypoellipticity of $G$, as we shall show in the sequel (see also [28], 29]. For instance, assume that condition (5.2) is violated at the origin for some $j_{0} \in \mathbb{N}$; namely,

$$
1+2 \alpha(0,0)=\frac{(-1)^{j_{0}+1}}{h}+(1+1 / h)\left(j_{0}+\theta\left(j_{0}\right)\right)
$$

and

$$
\partial_{x} \alpha(0,0)=0, \quad \partial_{x x}^{2} \alpha(0,0) \neq 0 .
$$

The above conditions on the derivatives ensure that $\alpha(x, y)$ is not constant and are chosen to make the exposition as clear as possible. 
The main result of this section is the following

Theorem 5.1. In the setting above, $G$ can be $C^{\infty}$-hypoelliptic with loss of $\delta \geq 2$ derivatives only. Moreover, assume that

$$
1+2 \alpha(0, y)\left\{\begin{array}{l}
\geq \frac{(-1)^{j_{0}+1}}{h}+(1+1 / h)\left(j_{0}+\theta\left(j_{0}\right)\right) \text { if } \partial_{x x}^{2} \alpha(0,0)>0 \\
\leq \frac{(-1)^{j_{0}+1}}{h}+(1+1 / h)\left(j_{0}+\theta\left(j_{0}\right)\right) \text { if } \partial_{x x}^{2} \alpha(0,0)<0
\end{array}\right.
$$

whenever $y$ is near 0 . Then $G$ is $C^{\infty}$-hypoelliptic with loss of derivatives exactly equal to 2 .

The last part of this section is devoted to the proof of the theorem above (we refer the reader to 28 for further details).

Let us now consider the "localized" operator $G_{y, \eta}$ at $(0, y, 0, \eta)$,

$$
G_{y, \eta}=D_{x}^{2}+x^{2 h} \eta^{2}-(1+2 \alpha(0, y)) h x^{h-1} \eta,
$$

and observe that $G_{y, \eta}$ is an anharmonic oscillator of type (1.1) by setting $a=\eta^{2}$ and $\mu=-(1+2 \alpha(0, y)) h \eta$; hence, its spectrum is discrete, made of simple eigenvalues

$$
\lambda_{0}(y, \eta)<\lambda_{1}(y, \eta)<\cdots<\lambda_{j}(y, \eta)<\lambda_{j+1}(y, \eta)<\cdots, \quad j \in \mathbb{N} .
$$

In view of Theorem 1.1. condition (5.4) implies that

$$
\lambda_{j_{0}}(0, \eta) \equiv 0 \quad \text { for } \quad \eta>0, \quad \text { whereas } \quad \lambda_{j_{0}}(0, \eta) \neq 0 \quad \text { for } \quad \eta<0 .
$$

Therefore, the localized operator (5.7) is not invertible at $y=0$, and this is actually the reason for which $G$ cannot be hypoelliptic with the loss of derivatives $2 h /(h+1)$.

Our aim is to show that $G$ can still be $C^{\infty}$-hypoelliptic under the hypothesis (5.6) on the behaviour of $\lambda_{j_{0}}(y, \eta)$ when $y$ is near zero. This will be achieved by constructing a sort of parametrix of $G$ starting from a "partial inverse" of its localized operator (5.7).

Let us start by giving a brief plan of the proof. The idea is essentially a linear algebra remark due to Schur (see [36]): assume that the $n \times n$ matrix $A$ has zero in its spectrum with multiplicity one. Then of course $A$ is not invertible, but, denoting by $e_{0}$ the zero eigenvector of $A$, the matrix (in block form)

$$
\left[\begin{array}{cc}
A & e_{0} \\
{ }^{t} e_{0} & 0
\end{array}\right]
$$

is invertible as an $(n+1) \times(n+1)$ matrix in $\mathbb{C}^{n+1}$. Here ${ }^{t} e_{0}$ denotes the row vector $e_{0}$.

In our case the localized operator $G_{y, \eta}$ has a zero simple eigenvalue and plays the role of the matrix $A$ above.

Denote by $\phi_{j_{0}}(x, y, \eta) \in C^{\infty}\left(\mathbb{R}_{(y, \eta)}^{2}, \mathcal{S}\left(\mathbb{R}_{x}\right)\right)$ a normalized eigenfunction of $G_{y, \eta}$ corresponding to the simple eigenvalue $\lambda_{j_{0}}(y, \eta)$. According to the above linear algebra remark, it is easy to see that the matrix operator

$$
\begin{gathered}
\mathcal{P}_{(y, \eta)}=\left(\begin{array}{cc}
G_{y, \eta} & \phi_{j_{0}}(\cdot, y, \eta) \\
\left\langle\cdot, \phi_{j_{0}}(\cdot, y, \eta)\right\rangle_{L^{2}\left(\mathbb{R}_{x}\right)} & 0
\end{array}\right): \mathcal{S}\left(\mathbb{R}_{x}\right) \times \mathbb{C} \longrightarrow \mathcal{S}\left(\mathbb{R}_{x}\right) \times \mathbb{C}, \\
\mathcal{P}_{(y, \eta)}(f(x), z)=\left(G_{y, \eta}(f)(x)+z \phi_{j_{0}}(x, y, \eta),\left\langle f, \phi_{j_{0}}(x, y, \eta)\right\rangle_{L^{2}\left(\mathbb{R}_{x}\right)}\right)
\end{gathered}
$$


is invertible for $y$ sufficiently near zero. The point is now to construct the following square system:

$\mathcal{P}=\left[\begin{array}{cc}G & \phi_{j_{0}}\left(x, y, D_{y}\right) \\ \phi_{j_{0}}^{*}\left(x, y, D_{y}\right) & 0\end{array}\right]: C_{0}^{\infty}\left(\mathbb{R}_{(x, y)}^{2}\right) \times C_{0}^{\infty}\left(\mathbb{R}_{y}\right) \longrightarrow C^{\infty}\left(\mathbb{R}_{(x, y)}^{2}\right) \times C^{\infty}\left(\mathbb{R}_{y}\right)$ in such a way that $\mathcal{P}_{(y, \eta)}$ is the "localized operator" naturally associated with $\mathcal{P}$ (all this procedure will be made rigorous in the sequel). Since $\mathcal{P}_{(y, \eta)}$ turns out to be invertible, we shall construct a parametrix $\mathcal{Q}$ of $\mathcal{P}$ having the form

$$
\mathcal{Q}=\left[\begin{array}{cc}
F\left(x, y, D_{x}, D_{y}\right) & \psi\left(x, y, D_{y}\right) \\
\psi^{*}\left(x, y, D_{y}\right) & -\Lambda\left(y, D_{y}\right)
\end{array}\right]
$$

where $\psi(\cdot, y, \eta) \in C^{\infty}\left(\mathbb{R}_{(y, \eta)}^{2}, \mathcal{S}\left(\mathbb{R}_{x}\right)\right), F\left(x, y, D_{x}, D_{y}\right)$ is constructed starting from a "partial" inverse of $G_{y, \eta}$ (see $F_{0}$ below in (5.23) ), and $\Lambda\left(y, D_{y}\right)$ is a pseudodifferential operator in the $y$-variable only, having $\lambda_{j_{0}}(y, \eta)$ as its principal symbol.

This idea was exploited by several authors in the study of hypoellipticity (see, for instance, [35, [15, and, in particular, the approach followed in 30]), and will allow us to reduce the problem of the hypoellipticity of $G$ to the simpler analysis of the one-variable pseudodifferential operator $\Lambda\left(y, D_{y}\right)$ (see, for instance, [5]).

Note that the operator $G$ is not transversally elliptic if $h>1$ (i.e., $G$ does not vanish exactly to the second order on its characteristic manifold $\Sigma=\{x=0=$ $\xi, \eta \neq 0\})$, due to

$$
\sigma_{\text {princ }}(G)=\xi^{2}+x^{2 h} \eta^{2} \approx \eta^{2}\left(|x|^{h}+\frac{|\xi|}{|\eta|}\right)^{2} .
$$

Therefore, in order to carry out the above program, we need a variant of the Boutet de Monvel pseudodifferential calculus (see 4 and also 15]), which is briefly recalled in Appendix II (further details can be found in Section 2 of [7] and in Appendix B of 28$]$. .

Let us now start the construction of a right parametrix $\mathcal{Q}$ of the operator $\mathcal{P}$ as a map from $C_{0}^{\infty}\left(\mathbb{R}_{(x, y)}^{2}\right) \times C_{0}^{\infty}\left(\mathbb{R}_{y}\right)$ into $C^{\infty}\left(\mathbb{R}_{(x, y)}^{2}\right) \times C^{\infty}\left(\mathbb{R}_{y}\right)$. A left parametrix $\mathcal{Q}^{\prime}$ of $\mathcal{P}$ can be easily obtained by arguing in a similar way, so that $\mathcal{Q}$ is actually a complete parametrix of $\mathcal{P}$ since, from standard pseudodifferential calculus, one trivially has $\mathcal{Q} \equiv \mathcal{Q}^{\prime}$ (mod. a regularizing operator).

Firstly, we introduce the pseudodifferential framework used to construct the matrix operator $\mathcal{P}$. To this aim, note that the eigenfunction

$$
\phi_{j_{0}}(\cdot, y, \eta) \in \operatorname{Ker}\left(G_{y, \eta}-\lambda_{j_{0}}(y, \eta) I d\right)
$$

is a rapidly decreasing function in $x$, smoothly depending on $(y, \eta)$, i.e., $\phi_{j_{0}}(\cdot, y, \eta) \in$ $C^{\infty}\left(\mathbb{R}_{(y, \eta)}^{2}, \mathcal{S}\left(\mathbb{R}_{x}\right)\right.$ ) (see, for instance, Thm. 1.9.1. and (1.9.2) in [16]). Furthermore, the symbol of the localized operator $G_{y, \eta}$ satisfies the scaling property

$$
\sigma\left(G_{y, \mu \eta}\right)\left(\mu^{-\frac{1}{h+1}} x, \mu^{\frac{1}{h+1}} \xi\right)=\mu^{2 /(h+1)} \sigma\left(G_{y, \eta}\right)(x, \xi),
$$

whence, the correspoding homogeneity properties immediately follow:

$$
\lambda_{j_{0}}(y, \mu \eta)=\mu^{2 /(h+1)} \lambda_{j_{0}}(y, \eta), \quad \phi_{j_{0}}\left(\mu^{-\frac{1}{h+1}} x, y, \mu \eta\right)=\mu^{1 / 2(h+1)} \phi_{j_{0}}(x, y, \eta), \quad \mu>0,
$$

where the factor $\mu^{1 / 2(h+1)}$ appears in the second formula because of the $L^{2}\left(\mathbb{R}_{x}\right)$ normalization of $\phi_{j_{0}}(\cdot, y, \eta)$. As a consequence, we get that $\lambda_{j_{0}}(y, \eta) \in \mathrm{S}_{1,0}^{2 /(h+1)}\left(\mathbb{R}^{2}\right)$ and $\phi_{j_{0}}(x, y, \eta) \in H_{h}^{1 / 2(h+1)}$ in view of (7.4) in Appendix II. 
We are looking for an operator $\mathcal{Q}$ such that

$$
\begin{array}{r}
{\left[\begin{array}{cc}
G\left(x, y, D_{x}, D_{y}\right) & \phi_{j_{0}}\left(x, y, D_{y}\right) \\
\phi_{j_{0}}^{*}\left(x, y, D_{y}\right) & 0
\end{array}\right] \circ\left[\begin{array}{cc}
F\left(x, y, D_{x}, D_{y}\right) & \psi\left(x, y, D_{y}\right) \\
\psi^{*}\left(x, y, D_{y}\right) & -\Lambda\left(y, D_{y}\right)
\end{array}\right]} \\
\equiv\left[\begin{array}{cc}
I d_{C_{0}^{\infty}\left(\mathbb{R}^{2}\right)} & 0 \\
0 & I d_{C_{0}^{\infty}(\mathbb{R})}
\end{array}\right]
\end{array}
$$

Here $\psi\left(x, y, D_{y}\right)$ and $\psi^{*}\left(x, y, D_{y}\right)$ denote operators in $O P H_{h}^{1 / 2(h+1)}$ (see Definition 7.3) and $O P H_{h}^{* 1 / 2(h+1)}$ (see Definition 7.4), $F \in O P S_{h}^{-2,-2}$ (see Definition 7.1) and $\Lambda \in O P S_{1,0}^{2 /(h+1)}$. Finally $\equiv$ means equality modulo a regularizing operator. From (5.10) we obtain the following four relations:

$$
\begin{aligned}
G\left(x, y, D_{x}, D_{y}\right) \circ F\left(x, y, D_{x}, D_{y}\right)+\phi_{j_{0}}\left(x, y, D_{y}\right) \circ \psi^{*}\left(x, y, D_{y}\right) & \equiv I d, \\
G\left(x, y, D_{x}, D_{y}\right) \circ \psi\left(x, y, D_{y}\right)-\phi_{j_{0}}\left(x, y, D_{y}\right) \circ \Lambda\left(y, D_{y}\right) & \equiv 0, \\
\phi_{j_{0}}^{*}\left(x, y, D_{y}\right) \circ F\left(x, y, D_{x}, D_{y}\right) & \equiv 0, \\
\phi_{j_{0}}^{*}\left(x, y, D_{y}\right) \circ \psi\left(x, y, D_{y}\right) & \equiv I d .
\end{aligned}
$$

We are going to find the symbols $F, \psi$ and $\Lambda$ as an asymptotic series of globally homogeneous symbols (see in Appendix II, Definition 7.5 and the corresponding notes below):

$$
F \sim \sum_{j \geq 0} F_{-j}, \quad \psi \sim \sum_{j \geq 0} \psi_{-j}, \quad \Lambda \sim \sum_{j \geq 0} \Lambda_{-j}
$$

In the sequel it is useful to write $G$ as an asymptotic sum of globally homogeneous functions; to this aim, by Taylor expanding the coefficient $\alpha(x, y)$ in (5.1) w.r.t. the $x$-variable at $x=0$, we get

$$
G\left(x, y, D_{x}, D_{y}\right)=\sum_{j \geq 0} G_{-j}\left(x, y, D_{x}, D_{y}\right)+O P S_{h}^{2, \infty},
$$

where

$$
\begin{aligned}
\sigma\left(G_{0}\right)(x, y, \xi, \eta) & =\xi^{2}+x^{2 h} \eta^{2}-(1+2 \alpha(0, y)) h x^{h-1} \eta, \\
\sigma\left(G_{-j}\right)(x, y, \xi, \eta) & =-\frac{2}{j !} \partial_{x}^{j} \alpha(0, y) h x^{j+h-1} \eta \quad \text { if } j>0 .
\end{aligned}
$$

According to Definition [7.5, the term $G_{-j}$ is the globally homogeneous part of degree $\frac{2}{h+1}-\frac{j}{h+1}$ of the symbol of $G$. Note that $G_{0}\left(x, y, D_{x}, \eta\right)$ coincides with the localized operator $G_{y, \eta}$ in (5.7); for this reason, the terms $G_{-j}\left(x, y, D_{x}, \eta\right)$ can be thought of as higher order localized operators of $G$.

By arguing as done in the proof of Prop. 3.1.2 of [15], in (5.10) we can indentify $G$ by its asymptotic sum $\sum_{j \geq 0} G_{-j}$, overlooking the error term in $O P S_{h}^{2, \infty}$.

From Lemma 7.1 we obtain that

$$
\sigma(G \circ F) \sim \sum_{s \geq 0} \sum_{(h+1) \alpha+i+j=s} \frac{1}{\alpha !} \sigma\left(\partial_{\eta}^{\alpha} G_{-j}\left(x, y, D_{x}, \eta\right) \circ_{x} D_{y}^{\alpha} F_{-i}\left(x, y, D_{x}, \eta\right)\right) .
$$

Furthermore from Lemma 7.2 (i) we may write that

$$
\sigma\left(\phi_{0} \circ \psi^{*}\right) \sim e^{-i x \xi} \sum_{s \geq 0} \sum_{(h+1) \alpha+i=s} \frac{1}{\alpha !} \partial_{\eta}^{\alpha} \phi_{j_{0}}(x, y, \eta) D_{y}^{\alpha} \hat{\bar{\psi}}_{-i}(\xi, y, \eta) .
$$


Analogously Lemmas 7.3, (7.2) (iii) give

$$
\begin{gathered}
\sigma(G \circ \psi) \sim \sum_{s \geq 0} \sum_{(h+1) \ell+i+j=s} \frac{1}{\ell !} \partial_{\eta}^{\ell} G_{-j}\left(x, y, D_{x}, \eta\right)\left(D_{y}^{\ell} \psi_{-i}(\cdot, y, \eta)\right), \\
\sigma\left(\phi_{j_{0}} \circ \Lambda\right) \sim \sum_{s \geq 0} \sum_{(h+1) \alpha+\ell=s} \frac{1}{\alpha !} \partial_{\eta}^{\alpha} \phi_{j_{0}}(x, y, \eta) D_{y}^{\alpha} \Lambda_{-\ell}(y, \eta) .
\end{gathered}
$$

Finally Lemmas 7.4(i) and 7.2(ii) yield

$$
\sigma\left(\phi_{j_{0}}^{*} \circ F\right) \sim \sum_{s \geq 0} \sum_{(h+1) \ell+j=s} \frac{1}{\ell !} D_{y}^{\ell}\left(\overline{F_{-j}}\left(x, y, D_{x}, \eta\right)\right)^{*}\left(\partial_{\eta}^{\ell} \overline{\phi_{j_{0}}}(\cdot, y, \eta)\right),
$$

and

$$
\sigma\left(\phi_{j_{0}}^{*} \circ \psi\right) \sim \sum_{s \geq 0} \sum_{(h+1) \alpha+j=s} \frac{1}{\alpha !} \int \partial_{\eta}^{\alpha} \bar{\phi}_{j_{0}}(x, y, \eta) D_{y}^{\alpha} \psi_{-j}(x, y, \eta) d x .
$$

Let us consider the terms globally homogeneous of degree 0 . We obtain the relations

$$
\begin{aligned}
G_{y, \eta}\left(x, y, D_{x}, \eta\right) \circ_{x} F_{0}\left(x, y, D_{x}, \eta\right)+\phi_{j_{0}}(x, y, \eta) \otimes \psi_{0}(\cdot, y, \eta) & =I d \\
G_{y, \eta}\left(x, y, D_{x}, \eta\right)\left(\psi_{0}(\cdot, y, \eta)\right)-\Lambda_{0}(y, \eta) \phi_{j_{0}}(x, y, \eta) & =0 \\
\left(F_{0}\left(x, y, D_{x}, \eta\right)\right)^{*}\left(\phi_{j_{0}}(\cdot, y, \eta)\right) & =0 \\
\int \bar{\phi}_{j_{0}}(x, y, \eta) \psi_{0}(x, y, \eta) d x & =1 .
\end{aligned}
$$

Here we denoted by $\phi_{j_{0}} \otimes \psi_{0}$ the operator $u=u(x) \mapsto \phi_{j_{0}} \int \bar{\psi}_{0} u d x ; \phi_{j_{0}} \otimes \psi_{0}$ must be a globally homogeneous symbol of degree zero.

By choosing

$$
\psi_{0}:=\phi_{j_{0}} \quad \text { and } \quad \Lambda_{0}(y, \eta):=\lambda_{j_{0}}(y, \eta)
$$

conditions (5.19) and (5.21) are automatically satisfied. Upon denoting by $\Pi_{0}$ the orthogonal projection onto $\operatorname{Ker}\left(G_{y, \eta}-\lambda_{j_{0}}(y, \eta) I d\right)=\left[\phi_{j_{0}}\right]$, Conditions (5.18) and (5.20) can be rewritten as

$$
\begin{aligned}
G_{y, \eta}\left(x, y, D_{x}, \eta\right) \circ_{x} F_{0}\left(x, y, D_{x}, \eta\right) & =I d-\Pi_{0}, \\
\operatorname{Im} F_{0}\left(x, y, D_{x}, \eta\right) & \subset\left[\phi_{j_{0}}\right]^{\perp},
\end{aligned}
$$

whence

$$
F_{0}\left(x, y, D_{x}, \eta\right)= \begin{cases}\left(G_{y,\left.\eta\right|_{\left[\phi_{j_{0}}\right] \perp \cap B_{h}}}\right)^{-1} & \text { on }\left[\phi_{j_{0}}\right]^{\perp}, \\ 0 & \text { on }\left[\phi_{j_{0}}\right]\end{cases}
$$

where $B_{h}=\left\{f \in L^{2}\left(\mathbb{R}_{x}\right) \mid x^{\alpha} \partial_{x}^{\beta} f \in L^{2}\left(\mathbb{R}_{x}\right), \quad \alpha / h+\beta \leq 2\right\}$. We point out that $G_{y, \eta}$ is $h$-globally elliptic w.r.t. $(x, \xi)$, smoothly depending on the parameters $(y, \eta)$, $\eta \neq 0$, i.e., for $|x|^{h}+|\xi| \gg 1$ one has that

$$
\left|\sigma\left(G_{y, \eta}\right)(x, \xi)\right|=\left|\xi^{2}+x^{2 h} \eta^{2}-(1+2 \alpha(0, y)) h x^{h-1} \eta\right| \geq C_{y, \eta}\left(|\xi|+|x|^{h}+1\right)^{2} .
$$

Following [16] one can show that $F_{0}\left(x, y, D_{x}, \eta\right)$ is actually a pseudodifferential operator whose symbol verifies (7.3) with $m=k=-2, j=0$, and is globally homogeneous of degree $-2 /(h+1)$. 
Once we have completed the analysis of the globally homogeneous terms of degree 0 in (5.10), let us pass to the terms of degree $-1 /(h+1)$ in Equations (5.11)-(5.14). Upon noting that $\frac{1}{h+1}<1$, we get

$$
\begin{aligned}
G_{-\frac{1}{h+1}} \circ_{x} F_{0}+G_{y, \eta} \circ_{x} F_{-\frac{1}{h+1}}+\phi_{j_{0}} \otimes \psi_{-\frac{1}{h+1}} & =0 \\
G_{y, \eta}\left(\psi_{-\frac{1}{h+1}}\right)+G_{-\frac{1}{h+1}}\left(\phi_{j_{0}}\right)-\Lambda_{-\frac{1}{h+1}} \phi_{j_{0}} & =0, \\
\left(F_{-\frac{1}{h+1}}\right)^{*}\left(\phi_{j_{0}}\right) & =0, \\
\left\langle\psi_{-\frac{1}{h+1}}, \phi_{j_{0}}\right\rangle_{L^{2}\left(\mathbb{R}_{x}\right)} & =0 .
\end{aligned}
$$

First we solve w.r.t. $\psi_{-\frac{1}{h+1}}$. From (5.27) we get immediately that $\psi_{-\frac{1}{h+1}} \in\left[\phi_{j_{0}}\right]^{\perp}$. (5.25) implies that

$$
G_{y, \eta}\left(\psi_{-\frac{1}{h+1}}\right)=-G_{-\frac{1}{h+1}}\left(\phi_{j_{0}}\right)+\Lambda_{-\frac{1}{h+1}} \phi_{j_{0}} .
$$

Thus, since $G_{y, \eta}\left(\left[\phi_{j_{0}}\right]^{\perp}\right) \subset\left[\phi_{j_{0}}\right]^{\perp}$ we must have that $G_{y, \eta}\left(\psi_{-\frac{1}{h+1}}\right) \in\left[\phi_{0}\right]^{\perp}$, whence

$$
\begin{gathered}
\Lambda_{-\frac{1}{h+1}}=\left\langle G_{-\frac{1}{h+1}}\left(\phi_{j_{0}}\right), \phi_{j_{0}}\right\rangle \\
\psi_{-\frac{1}{h+1}}=F_{0}\left(-G_{-\frac{1}{h+1}}\left(\phi_{j_{0}}\right)+\Lambda_{-\frac{1}{h+1}} \phi_{j_{0}}\right)=-F_{0}\left(G_{-\frac{1}{h+1}}\left(\phi_{j_{0}}\right)\right) .
\end{gathered}
$$

From (5.26) we deduce that $\Pi_{0} F_{-\frac{1}{h+1}} \equiv 0$. Then from (5.23), applying $F_{0}$ to both sides of (5.24), we obtain that

$$
F_{-\frac{1}{h+1}}=\left(I d-\Pi_{0}\right) F_{-\frac{1}{h+1}}=-F_{0}\left(G_{-\frac{1}{h+1}} \circ_{x} F_{0}+\phi_{j_{0}} \otimes \psi_{-\frac{1}{h+1}}\right) .
$$

Inspecting (5.29), (5.30) we see that $\psi_{-\frac{1}{h+1}} \in H_{h}^{\frac{1}{2(h+1)}-\frac{1}{(h+1)}}$, globally homogeneous of degree $1 / 2(h+1)-1 /(h+1)$, and $F_{-\frac{1}{h+1}} \in S_{h}^{-2,-2+\frac{1}{h}}$, globally homogeneous of degree $-2 /(h+1)-1 /(h+1)$. From (5.28) we have that $\Lambda_{-\frac{1}{h+1}}$ is a homogenous symbol in $S_{1,0}^{\frac{2}{h+1}-\frac{1}{h+1}}$. This ends the analysis of the terms of degree $-1 /(h+1)$ in (5.10).

Let us finally compute explicitly the terms of degree $-2 /(h+1)$, which will be useful in the sequel of this section. The procedure can be iterated for each level of homogeneity, arguing in a similar way.

Since $2 /(h+1)<1$, Equations (5.11)-(5.14) at level $-2 /(h+1)$ read

$$
\begin{aligned}
G_{-\frac{2}{h+1}} \circ_{x} F_{0}+G_{-\frac{1}{h+1}} \circ_{x} F_{-\frac{1}{h+1}}+G_{y, \eta} \circ_{x} F_{-\frac{2}{h+1}}+\phi_{j_{0}} \otimes \psi_{-\frac{2}{h+1}} & =0 \\
G_{y, \eta}\left(\psi_{-\frac{2}{h+1}}\right)+G_{-\frac{1}{h+1}}\left(\psi_{-\frac{1}{h+1}}\right)+G_{-\frac{2}{h+1}}\left(\phi_{j_{0}}\right)-\Lambda_{-\frac{2}{h+1}} \phi_{j_{0}} & =0 \\
\left(F_{-\frac{2}{h+1}}\right)^{*}\left(\phi_{j_{0}}\right) & =0 \\
\left\langle\psi_{-\frac{2}{h+1}}, \phi_{j_{0}}\right\rangle_{L^{2}\left(\mathbb{R}_{x}\right)} & =0 .
\end{aligned}
$$

Arguing as above, from (5.34) we have that $\psi_{-\frac{2}{h+1}} \in\left[\phi_{j_{0}}\right]^{\perp}$, so that (5.32) gives that

$$
\left[\phi_{j_{0}}\right]^{\perp} \ni G_{y, \eta}\left(\psi_{-\frac{2}{h+1}}\right)=-G_{-\frac{1}{h+1}}\left(\psi_{-\frac{1}{h+1}}\right)-G_{-\frac{2}{h+1}}\left(\phi_{j_{0}}\right)+\Lambda_{-\frac{2}{h+1}} \phi_{j_{0}}
$$


whence, from (5.29) it follows that

$$
\begin{aligned}
\Lambda_{-\frac{2}{h+1}} & =\left\langle G_{-\frac{2}{h+1}}\left(\phi_{j_{0}}\right), \phi_{j_{0}}\right\rangle+\left\langle G_{-\frac{1}{h+1}}\left(\psi_{-\frac{1}{h+1}}\right), \phi_{j_{0}}\right\rangle \\
& =\left\langle G_{-\frac{2}{h+1}}\left(\phi_{j_{0}}\right), \phi_{j_{0}}\right\rangle-\left\langle F_{0}\left(G_{-\frac{1}{h+1}}\left(\phi_{j_{0}}\right)\right), G_{-\frac{1}{h+1}}^{*}\left(\phi_{j_{0}}\right)\right\rangle
\end{aligned}
$$

and that

$$
\psi_{-\frac{2}{h+1}}=F_{0}\left(-G_{-\frac{1}{h+1}}\left(\psi_{-\frac{1}{h+1}}\right)-G_{-\frac{2}{h+1}}\left(\phi_{j_{0}}\right)+\Lambda_{-\frac{2}{h+1}} \phi_{j_{0}}\right) .
$$

In view of (5.33) we see that $\Pi_{0} F_{-\frac{2}{h+1}} \equiv 0$. As a consequence, applying $F_{0}$ to both sides of (5.31) yields that

$$
F_{-\frac{2}{h+1}}=-F_{0}\left(G_{-\frac{2}{h+1}} \circ_{x} F_{0}+G_{-\frac{1}{h+1}} \circ_{x} F_{-\frac{1}{h+1}}+\phi_{j_{0}} \otimes \psi_{-\frac{2}{h+1}}\right) .
$$

This completes the proof of (5.10). Mirroring the argument above, we see that $\mathcal{Q}$ is also a left parametrix,

$$
\begin{array}{r}
{\left[\begin{array}{cc}
F\left(x, y, D_{x}, D_{y}\right) & \psi\left(x, y, D_{y}\right) \\
\psi^{*}\left(x, y, D_{y}\right) & -\Lambda\left(y, D_{y}\right)
\end{array}\right] \circ\left[\begin{array}{cc}
G\left(x, y, D_{x}, D_{y}\right) & \phi_{j_{0}}\left(x, y, D_{y}\right) \\
\phi_{j_{0}}^{*}\left(x, y, D_{y}\right) & 0
\end{array}\right]} \\
\equiv\left[\begin{array}{cc}
I d_{C_{0}^{\infty}\left(\mathbb{R}^{2}\right)} & 0 \\
0 & I d_{C_{0}^{\infty}(\mathbb{R})}
\end{array}\right]
\end{array}
$$

so that

$$
\begin{aligned}
F\left(x, y, D_{x}, D_{y}\right) \circ G\left(x, y, D_{x}, D_{y}\right) & =I d-\psi\left(x, y, D_{y}\right) \circ \phi_{j_{0}}^{*}\left(x, y, D_{y}\right), \\
\psi^{*}\left(x, y, D_{y}\right) \circ G\left(x, y, D_{x}, D_{y}\right) & =\Lambda\left(y, D_{y}\right) \circ \phi_{j_{0}}^{*}\left(x, y, D_{y}\right) .
\end{aligned}
$$

As a consequence, we have the following

Proposition 5.2. If $\Lambda$ is hypoelliptic with a loss of $\delta>0$ derivatives, then $G$ is also hypoelliptic with a loss of derivatives equal to $2 \frac{h}{h+1}+\delta$.

Proof. Assume that $G u \in H_{l o c}^{s}\left(\mathbb{R}^{2}\right)$. From Lemma 7.5 we have that $F G u \in$ $H_{l o c}^{s+2 /(h+1)}\left(\mathbb{R}^{2}\right)$. By (5.37) we have that $u-\psi \phi_{j_{0}}^{*} u \in H_{l o c}^{s+2 /(h+1)}\left(\mathbb{R}^{2}\right)$. Again, using Lemma 7.5] $\psi^{*} G u \in H_{l o c}^{s}(\mathbb{R})$, so that, by (5.38), $\Lambda \phi_{j_{0}}^{*} u \in H_{l o c}^{s}(\mathbb{R})$. The hypoellipticity of $\Lambda$ then yields that $\phi_{j_{0}}^{*} u \in H_{l o c}^{s+\frac{2}{h+1}-\delta}(\mathbb{R})$. From Lemma 7.5 we obtain that $\psi \phi_{j_{0}}^{*} u \in H_{l o c}^{s+\frac{2}{h+1}-\delta}(\mathbb{R})$. Thus $u=\left(I d-\psi \phi_{j_{0}}^{*}\right) u+\psi \phi_{j_{0}}^{*} u \in H_{l o c}^{s+\frac{2}{h+1}-\delta}=$ $H_{l o c}^{s+2-\frac{2 h}{h+1}-\delta}$. This proves the proposition.

Remark 5.3. The converse of Proposition 5.2 is also true and can be shown similarly by using the identities (5.12) and (5.14).

By applying Proposition 5.2 we have reduced the study of the hypoellipticity (h.e.) of $G$ to the analysis of the h.e. of $\Lambda\left(y, D_{y}\right) \sim \sum_{j>0} \Lambda_{-j}\left(y, D_{y}\right)$ in (5.15).

Let us now write explicitly the symbol $\Lambda(y, \eta)$, by taking into account (5.22), (5.28), (5.35) and (5.17):

$$
\Lambda_{0}(y, \eta)=\lambda_{j_{0}}(y, \eta)
$$

$$
\Lambda_{-\frac{1}{h+1}}=\left\langle G_{-\frac{1}{h+1}}\left(\phi_{j_{0}}\right), \phi_{j_{0}}\right\rangle=-2 \partial_{x} \alpha(0, y) h \eta\left\langle x^{h} \phi_{j_{0}}, \phi_{j_{0}}\right\rangle
$$

$$
\Lambda_{-\frac{2}{h+1}}=\left\langle G_{-\frac{2}{h+1}}\left(\phi_{j_{0}}\right), \phi_{j_{0}}\right\rangle-\left\langle F_{0}\left(G_{-\frac{1}{h+1}}\left(\phi_{j_{0}}\right),\right), G_{-\frac{1}{h+1}}^{*}\left(\phi_{j_{0}}\right)\right\rangle
$$

$$
=-\partial_{x x}^{2} \alpha(0, y) h \eta\left\langle x^{h+1} \phi_{j_{0}}, \phi_{j_{0}}\right\rangle-4 \partial_{x} \alpha(0, y)^{2} h^{2} \eta^{2}\left\langle F_{0}\left(x^{h} \phi_{j_{0}}\right), x^{h} \phi_{j_{0}}\right\rangle \text {. }
$$


We are going to show that $\Lambda_{-\frac{1}{h+1}}(y, \eta)$ vanishes identically. To see this, we recall that $h$ is an odd integer, hence the localized operator $G_{y, \eta}$ in (5.7) is invariant under the action of the antipodal map $x \longrightarrow-x$. Since the eigenvalue $\lambda_{j_{0}}(y, \eta)$ is simple (see the Introduction), the eigenfunction $\phi_{j_{0}}$ must have a parity with respect to the $x$ variable, so that $\left\langle x^{h} \phi_{j_{0}}, \phi_{j_{0}}\right\rangle \equiv 0$, and then

$$
\Lambda_{-\frac{1}{h+1}}(y, \eta) \equiv 0 \text {. }
$$

Moreover, due to (5.8) one also has that

$$
\Lambda_{0}(0, \eta)=\lambda_{j_{0}}(0, \eta) \equiv 0 \quad \text { for } \quad \eta>0 .
$$

From Lemma 27.1.5, Vol. IV of [22], the h.e. of $\Lambda=\Lambda\left(y, D_{y}\right) \in O P S_{1,0}^{2 /(h+1)}$ with loss of $0<\delta<1$ derivatives (near the origin) is equivalent to the following a priori estimate:

$$
\|u\|_{\frac{2}{h+1}-\delta}^{2} \leq C\left(\|\Lambda u\|_{0}^{2}+\|u\|_{\mu}^{2}\right), \quad \forall u \in C_{0}^{\infty}(K),
$$

where $K$ is a compact neighborhood of the origin and $\mu<2 /(h+1)-\delta$.

Firstly, we observe that $\Lambda(y, \eta) \equiv \lambda_{j_{0}}(y, \eta) \quad\left(\bmod . O P S_{1,0}^{2 /(h+1)-\delta}\right)$ whenever $\delta<2 /(h+1)$, and hence $\Lambda(0, \eta) \equiv 0\left(\bmod . O P S_{1,0}^{2 /(h+1)-\delta}\right)$ for every $\eta>0$. As a consequence of a standard localization argument, (5.41) cannot hold if $\delta<2 /(h+1)$; therefore, due to Lemma 27.1.5, Vol. IV of [22], $\Lambda\left(y, D_{y}\right)$ can be h.e. with loss of $\delta \geq 2 /(h+1)$ only, and by Remark 5.3 the same holds for $G$ with the minimal loss of derivatives equal to $2 h /(h+1)+2 /(h+1)=2$. This proves the first claim of Theorem 5.1.

We are left to show that, under the assumption (5.6), $G$ is h.e. with loss of 2 derivatives, which means, via Proposition 5.2 , that $\Lambda\left(y, D_{y}\right)$ is h.e. with loss of $2 /(h+1)$ derivatives. As observed above, we are reduced to prove (5.41) with $\delta=2 /(h+1)$. In doing this, we shall assume that $\eta>0$; indeed, if $\eta<0, \Lambda\left(y, D_{y}\right)$ is elliptic because of (5.39) and (5.8). This task will be achieved by applying the Fefferman-Phong inequality (see [10] and also Thm. 18.6.8, Vol. III of 22]) to estimate the quadratic form $\|\Lambda u\|_{0}^{2}=\left\langle\Lambda^{*} \Lambda u, u\right\rangle$. To this aim it is convenient to use the Weyl quantization (see Section 18.5, Vol. III of [22]), and to point out that the terms $\Lambda_{-j /(h+1)}(y, \eta)$ in the asymptotic expansion of $\Lambda \sim \sum_{j \geq 0} \Lambda_{-j /(h+1)}$ are homogeneous of order $2 /(h+1)-j /(h+1)$. By Theorem 18.5.10, Vol. III of [22] and by (5.39) we get

$$
\sigma^{w}(\Lambda)=\lambda_{j_{0}}(y, \eta)+\Lambda_{-\frac{2}{h+1}}(y, \eta)+\frac{i}{2} \partial_{\eta} \partial_{y} \lambda_{j_{0}}(y, \eta)+e(y, \eta), \quad \sigma^{w}\left(\Lambda^{*}\right)=\overline{\sigma^{w}(\Lambda)}
$$

where $e(y, \eta) \in S_{1,0}^{-\frac{1}{h+1}}$. We recall that, in our case, $h>1$ is an odd integer, thus $h$ is actually equal or greater than 3 . The Weyl pseudodifferential calculus (see Thm. 18.5.4, Vol. III of [22]) yields

$$
\begin{gathered}
\sigma^{w}\left(\Lambda^{*} \Lambda\right)=\lambda_{j_{0}}^{2}+2 \lambda_{j_{0}} \Lambda_{-\frac{2}{h+1}}+\Lambda_{-\frac{2}{h+1}}^{2}+2 \lambda_{j_{0}} \operatorname{Re} e+e^{\prime} \\
\geq\left(1-\epsilon^{2}\right) \lambda_{j_{0}}^{2}+2 \lambda_{j_{0}} \Lambda_{-\frac{2}{h+1}}+\Lambda_{-\frac{2}{h+1}}^{2}+e^{\prime \prime}
\end{gathered}
$$

where $e^{\prime}(y, \eta)$ is an error term of order $-\frac{1}{h+1}, e^{\prime \prime}:=-1 / \epsilon^{2}(\operatorname{Re} e)^{2}+e^{\prime} \in S_{1,0}^{-\frac{1}{h+1}}$ and $\epsilon$ is a fixed positive constant less than 1 . 
The key point is to recognize that, under the hypotheses of Theorem 5.1 ,

$$
\lambda_{j_{0}}(y, \eta) \Lambda_{-\frac{2}{h+1}}(y, \eta) \geq 0
$$

whenever $y$ is sufficiently near zero. From (5.5) and (5.40) it follows that

$$
\begin{aligned}
\Lambda_{-\frac{2}{h+1}}(0, \eta) & =-\partial_{x x}^{2} \alpha(0,0) h \eta\left\langle x^{h+1} \phi_{j_{0}}, \phi_{j_{0}}\right\rangle_{\mid y=0} \\
& =-\partial_{x x}^{2} \alpha(0,0) h \eta\left\|x^{\frac{h+1}{2}} \phi_{j_{0}}\right\|_{\mid y=0}^{2} \neq 0 .
\end{aligned}
$$

Here we have that $\eta>0$. Hence, due to (5.9) the condition $\lambda_{j_{0}}(y, \eta) \Lambda_{-\frac{2}{h+1}}(y, \eta) \geq 0$ means that

$$
\lambda_{j_{0}}(y, 1) \leq 0 \text { if } \partial_{x x}^{2} \alpha(0,0)>0 \quad \text { and } \quad \lambda_{j_{0}}(y, 1) \geq 0 \text { if } \partial_{x x}^{2} \alpha(0,0)<0 .
$$

We stress that the "true" value of the eigenvalue $\lambda_{j_{0}}(y, 1)$ is a priori unknown; nevertheless, by virtue of Theorem 1.1. conditions (5.43) can be explicitly read from the operator $G$ and they are equivalent to hypothesis (5.6) of Theorem 5.1.

Therefore, in (5.42) we find out that, for $y$ near zero,

$$
\sigma^{w}\left(\Lambda^{*} \Lambda\right)(y, \eta) \geq \Lambda_{-\frac{2}{h+1}}(y, \eta)^{2}+e^{\prime \prime}(y, \eta) \geq \theta+e^{\prime \prime}(y, \eta),
$$

where $\theta$ is a suitable positive constant.

Finally, by applying the Fefferman-Phong Inequality to $\Lambda^{*} \Lambda-\theta I-\mathrm{Op}^{w}\left(e^{\prime \prime}\right)$ we finally get

$$
\begin{aligned}
\|\Lambda u\|_{0}^{2}= & \left\langle\Lambda^{*} \Lambda u, u\right\rangle \geq \theta\|u\|_{0}^{2}+\left\langle\mathrm{Op}^{w}\left(e^{\prime \prime}\right) u, u\right\rangle-C\|u\|_{\frac{4}{h+1}-2}^{2} \\
& \geq \theta\|u\|_{0}^{2}-C^{\prime}\|u\|_{-\frac{1}{2(h+1)}}^{2}-C\|u\|_{\frac{4}{h+1}-2}^{2},
\end{aligned}
$$

which is exactly the inequality (5.41) with $\delta=2 /(h+1)$, that we were seeking.

Note that the full strength of the Fefferman-Phong Inequality is actually used in the case $h=3$ only; as a matter of fact, for the other values of $h>3$, the Sharp Gårding Inequality (see Thm. 18.1.14, Vol. III of [22]) provides the correct estimate.

Finally, we point out that, if $h>3$, a parametrix of $\Lambda\left(y, D_{y}\right)$ can be constructed by noting, as seen above, that $|\sigma(\Lambda)(y, \eta)| \geq c>0(|\eta| \gg 1)$, and thus using Thm. 22.1.3, Vol. III of 22].

\section{Appendix I: Perturbation theory for the Anharmonic oscillator $P_{\mu}$}

In this appendix, for the sake of completeness, we recall some classical results about the Rayleigh-Schrödinger series applied to our anharmonic oscillator $P_{\mu}$. As an immediate consequence, we shall show that the eigenfunctions and the eigenvalues of $P_{\mu}$ depend smoothly on the parameter $\mu$.

Fix $\bar{\mu} \in \mathbb{R}$ and let us write, for simplicity,

$$
P_{\mu}=-\partial_{x}^{2}+a x^{2 h}+\mu x^{h-1}=H_{0}+\epsilon V,
$$

where $H_{0}=-\partial_{x}^{2}+a x^{2 h}+\bar{\mu} x^{h-1}, \epsilon=\mu-\bar{\mu}$, and $V=x^{h-1}$. We trivially have that the domains of the operators $H_{0}$ and $V$ satisfy the relation $\mathcal{D}\left(H_{0}\right)=B_{h} \subset \mathcal{D}(V)$ 
(see (1.2)); furthermore, since $x^{2 h}-x^{2 h-2}+1 \geq 0$, we easily see that, for all $\psi \in B_{h}$,

$$
\begin{aligned}
\frac{1}{a}\left\langle H_{0} \psi, \psi\right\rangle+\left(1+\bar{\mu}^{2} / a^{2}\right)\|\psi\|_{0}^{2} & \geq\left\langle\left(x^{2 h}+(\bar{\mu} / a) x^{h-1}+1+\bar{\mu}^{2} / a^{2}\right) \psi, \psi\right\rangle \\
& \geq\left\langle\left(x^{2 h-2}+(\bar{\mu} / a) x^{h-1}+\bar{\mu}^{2} / a^{2}\right) \psi, \psi\right\rangle \\
& =\left\langle\left(3 / 4 x^{2 h-2}+\left(1 / 2 x^{h-1}+\bar{\mu} / a\right)^{2}\right) \psi, \psi\right\rangle \\
& \geq 3 / 4\left\|x^{h-1} \psi\right\|_{0}^{2},
\end{aligned}
$$

whence, by the Cauchy-Schwarz inequality one gets

$$
\frac{1}{a}\left\|H_{0} \psi\right\|_{0}^{2}+\left(1+\bar{\mu}^{2} / a^{2}+\frac{1}{a}\right)\|\psi\|_{0}^{2} \geq 3 / 4\|V \psi\|_{0}^{2} .
$$

As a result, by the lemma in Chapter XII.2 of [32], p. 16, the oscillator $H_{0}+\epsilon V$, thought of as an unbounded operator in $L^{2}\left(\mathbb{R}_{x}\right)$ with domain $B_{h}$, is an analytic family in the sense of Kato (analytic family of type $(A)$ ).

By Proposition 3.3, Chap. 2 of [1], $H_{0}=P_{\bar{\mu}}$ has a discrete spectrum made of the simple eigenvalues $\lambda_{j}(\bar{\mu})$. If we fix $j_{0} \in \mathbb{N}$, there exists a $\delta=\delta\left(j_{0}\right)>0$, small enough, such that the operator

$$
\Pi_{\epsilon}=\frac{1}{2 \pi i} \oint_{\left|\sigma-\lambda_{j_{0}}(\bar{\mu})\right|=\delta}\left(\sigma I-H_{0}-\epsilon V\right)^{-1} d \sigma
$$

is the orthogonal projection onto the eigenspace $\operatorname{Ker}\left(H_{0}+\epsilon V-\lambda_{j_{0}}(\bar{\mu}+\epsilon) I\right)$, provided that $\epsilon=\mu-\bar{\mu}$ is small enough (see, for instance, Theorem XII.6 and XII.8 of [32]).

We have that

$$
\sigma I-H_{0}-\epsilon V=\left(I-\epsilon V\left(\sigma I-H_{0}\right)^{-1}\right)\left(\sigma I-H_{0}\right),
$$

whence

$$
\left(\sigma I-H_{0}-\epsilon V\right)^{-1}=\left(\sigma I-H_{0}\right)^{-1}\left(I-\epsilon V\left(\sigma I-H_{0}\right)^{-1}\right)^{-1} .
$$

In view of (6.1), $V\left(\sigma I-H_{0}\right)^{-1}$ is bounded (see Theorem XII.9 32 for further details); thus an application of the Neumann series shows that $\Pi_{\epsilon}$ is analytic in $\epsilon$ near the origin. Precisely, we obtain

$$
\left(\sigma I-H_{0}-\epsilon V\right)^{-1}=\sum_{s=0}^{+\infty} \epsilon^{s}\left(\sigma I-H_{0}\right)^{-1}\left(V\left(\sigma I-H_{0}\right)^{-1}\right)^{s}
$$

whence

$$
\Pi_{\epsilon}=\sum_{s=0}^{+\infty} \epsilon^{s} A_{s}, \quad A_{s}=\frac{1}{2 \pi i} \oint_{\left|\sigma-\lambda_{j_{0}}(\bar{\mu})\right|=\delta}\left(\sigma I-H_{0}\right)^{-1}\left(V\left(\sigma I-H_{0}\right)^{-1}\right)^{s} d \sigma
$$

Let $\phi_{j_{0}}(x) \in L^{2}(\mathbb{R})$ be a real normalized eigenfunction corresponding to the eigenvalue $\lambda_{j_{0}}(\bar{\mu})$; we obtain that $\Pi_{\epsilon} \phi_{j_{0}}(x) \in \operatorname{Ker}\left(H_{0}+\epsilon V-\lambda_{j_{0}}(\bar{\mu}+\epsilon) I\right)$ is analytic in $\epsilon$ and $\left\|\Pi_{\epsilon} \phi_{j_{0}}\right\|_{L^{2}(\mathbb{R})}^{2}=\left\|\phi_{j_{0}}\right\|_{L^{2}(\mathbb{R})}^{2}=1$. Due to (6.3), the analyticity of the eigenvalue $\lambda_{j_{0}}(\bar{\mu}+\epsilon)$ is a consequence of the following identities:

$$
\begin{aligned}
\lambda_{j_{0}}(\bar{\mu}+\epsilon) & =\lambda_{j_{0}}(\bar{\mu}+\epsilon)\left\|\Pi_{\epsilon} \phi_{j_{0}}\right\|_{L^{2}(\mathbb{R})}^{2} \\
& =\lambda_{j_{0}}(\bar{\mu}+\epsilon)\left\langle\Pi_{\epsilon} \phi_{j_{0}}, \phi_{j_{0}}\right\rangle=\left\langle\left(H_{0}+\epsilon V\right) \Pi_{\epsilon} \phi_{j_{0}}, \phi_{j_{0}}\right\rangle \\
& =\left\langle\Pi_{\epsilon} \phi_{j_{0}}, H_{0} \phi_{j_{0}}\right\rangle+\epsilon\left\langle V \Pi_{\epsilon} \phi_{j_{0}}, \phi_{j_{0}}\right\rangle=\lambda_{j_{0}}(\bar{\mu})+\left\langle\epsilon V \Pi_{\epsilon} \phi_{j_{0}}, \phi_{j_{0}}\right\rangle .
\end{aligned}
$$


Precisely, by inserting (6.3) into the above formula, we get the so-called RayleighSchrödinger series for the eigenvalue $\lambda_{j_{0}}$ :

$$
\lambda_{j_{0}}(\bar{\mu}+\epsilon)=\lambda_{j_{0}}(\bar{\mu})+\sum_{s=0}^{+\infty} \epsilon^{s+1}\left\langle V A_{s} \phi_{j_{0}}, \phi_{j_{0}}\right\rangle .
$$

In particular, it will be useful to have explicit formulas for the first and the second derivative of $\lambda_{j_{0}}$ at $\bar{\mu}$.

To this purpose, we observe that

$$
A_{0} \phi_{j_{0}}=\frac{1}{2 \pi i} \oint_{\left|\sigma-\lambda_{j_{0}}(\bar{\mu})\right|=\delta}\left(\sigma I-H_{0}\right)^{-1} d \sigma \phi_{j_{0}}=\Pi_{0} \phi_{j_{0}}=\phi_{j_{0}}
$$

and we immediately get that

$$
\lambda_{j_{0}}^{\prime}(\bar{\mu})=\left\langle V \phi_{j_{0}}, \phi_{j_{0}}\right\rangle,
$$

as shown in (2.3) by following a different approach.

Furthermore, by (6.3) we have that

$$
\begin{aligned}
& \lambda_{j_{0}}^{\prime \prime}(\bar{\mu})=2\left\langle V A_{1} \phi_{j_{0}}, \phi_{j_{0}}\right\rangle=\frac{1}{\pi i} \oint_{\left|\sigma-\lambda_{j_{0}}(\bar{\mu})\right|=\delta}\left\langle\left(\sigma I-H_{0}\right)^{-1} V\left(\sigma I-H_{0}\right)^{-1} \phi_{j_{0}}, V \phi_{j_{0}}\right\rangle d \sigma \\
& (6.7) \quad=\frac{1}{\pi i} \oint_{\left|\sigma-\lambda_{j_{0}}(\bar{\mu})\right|=\delta}\left(\sigma-\lambda_{j_{0}}(\bar{\mu})\right)^{-1}\left\langle\left(\sigma I-H_{0}\right)^{-1} V \phi_{j_{0}}, V \phi_{j_{0}}\right\rangle d \sigma .
\end{aligned}
$$

From Theorem 3.1, Chap. 2 of 1, the eigenfunctions of the Schrödinger operator give rise to a complete orthonormal system $\left\{\phi_{j}\right\}_{j \in \mathbb{N}}$ of $L^{2}(\mathbb{R})$; we thus have that $V \phi_{j_{0}}=\sum_{n=0}^{+\infty}\left\langle V \phi_{j_{0}}, \phi_{n}\right\rangle \phi_{n}$ and that

$$
\left(\sigma I-H_{0}\right)^{-1} V \phi_{j_{0}}=\sum_{n=0}^{+\infty}\left\langle V \phi_{j_{0}}, \phi_{n}\right\rangle\left(\sigma I-H_{0}\right)^{-1} \phi_{n}=\sum_{n=0}^{+\infty}\left\langle V \phi_{j_{0}}, \phi_{n}\right\rangle\left(\sigma-\lambda_{n}(\bar{\mu})\right)^{-1} \phi_{n} .
$$

By using this identity in (6.7), we obtain

$$
\begin{aligned}
\lambda_{j_{0}}^{\prime \prime}(\bar{\mu}) & =\sum_{n=0}^{+\infty} \frac{1}{\pi i} \oint_{\left|\sigma-\lambda_{j_{0}}(\bar{\mu})\right|=\delta}\left(\sigma-\lambda_{j_{0}}(\bar{\mu})\right)^{-1}\left(\sigma-\lambda_{n}(\bar{\mu})\right)^{-1}\left\langle V \phi_{j_{0}}, \phi_{n}\right\rangle\left\langle\phi_{n}, V \phi_{j_{0}}\right\rangle d \sigma \\
& =\sum_{n=0}^{+\infty} 2\left|\left\langle V \phi_{j_{0}}, \phi_{n}\right\rangle\right|^{2} \underbrace{\frac{1}{2 \pi i} \oint_{\left|\sigma-\lambda_{j_{0}}(\bar{\mu})\right|=\delta}\left(\sigma-\lambda_{j_{0}}(\bar{\mu})\right)^{-1}\left(\sigma-\lambda_{n}(\bar{\mu})\right)^{-1} d \sigma .}_{=: C_{j_{0}, n}}
\end{aligned}
$$

We trivially have that $C_{j_{0}, j_{0}}=0$, whereas, if $n \neq j_{0}$, we see that

$$
\begin{aligned}
C_{j_{0}, n}=\frac{1}{\lambda_{j_{0}}(\bar{\mu})-\lambda_{n}(\bar{\mu})}\left(\frac{1}{2 \pi i} \oint_{\left|\sigma-\lambda_{j_{0}}(\bar{\mu})\right|=\delta}\right. & \frac{1}{\sigma-\lambda_{j_{0}}(\bar{\mu})} d \sigma \\
& \left.-\frac{1}{2 \pi i} \oint_{\left|\sigma-\lambda_{j_{0}}(\bar{\mu})\right|=\delta} \frac{1}{\sigma-\lambda_{n}(\bar{\mu})} d \sigma\right) .
\end{aligned}
$$

The above integrals represent the index of the point $\lambda_{j_{0}}(\bar{\mu})$ and $\lambda_{n}(\bar{\mu})$ with respect to the circle $\left|\sigma-\lambda_{j_{0}}(\bar{\mu})\right|=\delta$; since $\lambda_{j_{0}}(\bar{\mu})$ lies inside the circle, whereas $\lambda_{n}(\bar{\mu})$ lies 
outside, we obtain that $C_{j_{0}, n}=\frac{1}{\lambda_{j_{0}}(\bar{\mu})-\lambda_{n}(\bar{\mu})}$, whence we get

$$
\lambda_{j_{0}}^{\prime \prime}(\bar{\mu})=\sum_{\substack{n \\ n \neq j_{0}}}^{+\infty} \frac{2\left|\left\langle V \phi_{j_{0}}, \phi_{n}\right\rangle\right|^{2}}{\lambda_{j_{0}}(\bar{\mu})-\lambda_{n}(\bar{\mu})}
$$

In particular, if we choose $j_{0}=0$, i.e., the lowest eigenvalue, we have that $\lambda_{0}(\bar{\mu})-$ $\lambda_{n}(\bar{\mu})<0$ for $n \geq 1$, which yields that

$$
\lambda_{0}^{\prime \prime}(\bar{\mu})=\sum_{n=1}^{+\infty} \frac{2\left|\left\langle V \phi_{0}, \phi_{n}\right\rangle\right|^{2}}{\lambda_{0}(\bar{\mu})-\lambda_{n}(\bar{\mu})} \leq 0 .
$$

As a matter of fact, the above inequality is actually strict. To see this, assume, for the sake of argument, that $\lambda_{0}^{\prime \prime}(\bar{\mu})=0$. From (6.9) it would follow that $\left\langle V \phi_{0}, \phi_{n}\right\rangle=0$ for every $n \geq 1$, and hence that $V \phi_{0}=x^{h-1} \phi_{0} \in\left\langle\phi_{1}, \phi_{2}, \ldots\right\rangle^{\perp}=\left\langle\phi_{0}\right\rangle$, which is false $(h>1)$. Therefore, we have proved that

$$
\lambda_{0}^{\prime \prime}(\mu)<0, \quad \forall \mu \in \mathbb{R} .
$$

As a result, the lowest eigenvalue $\lambda_{0}(\mu)$ is a strictly concave function of $\mu \in \mathbb{R}$.

\section{Appendix II: A variant of the Boutet de Monvel Calculus}

In this appendix, following the ideas of 3 and 4, we develop the Boutet de Monvel Calculus adapted to our non-transversally elliptic case (further details can be found in Section 4 of [6] and in Appendix B of [28]). Firstly, we introduce the classes of symbols and operators we deal with; essentially, they are obtained by looking at $G$ in (5.1) (see Definition 7.1 below) and at the eigenfunctions of its localized operator (5.7) (see Definition 7.3). Finally we discuss their corresponding composition formulas.

Definition 7.1. We define the class of symbols $S_{h}^{m, k}(\Omega, \Sigma)$, where $\Omega$ is a conic neighborhood of the point $\left(0, e_{2}\right)$ and $\Sigma$ denotes the characteristic manifold $\{x=$ $0, \xi=0\}$, as the set of all $C^{\infty}$ functions such that, on any conic subset of $\Omega$ with compact base,

$$
\left|\partial_{y}^{\alpha} \partial_{\eta}^{\beta} \partial_{x}^{\gamma} \partial_{\xi}^{\delta} a(x, y, \xi, \eta)\right| \lesssim(1+|\eta|)^{m-\beta-\delta}\left(\frac{|\xi|}{|\eta|}+|x|^{h}+\frac{1}{|\eta|^{\frac{h}{h+1}}}\right)^{k-\frac{\gamma}{h}-\delta} .
$$

We simply write $S_{h}^{m, k}$ for $S_{h}^{m, k}\left(\mathbb{R}^{2} \times \mathbb{R}^{2}, \Sigma\right)$ and we set $S_{h}^{m, \infty}=\bigcap_{j \geq 0} S_{h}^{m, j}$. Finally we denote by $O P S_{h}^{m, k}$ and by $O P S_{h}^{m, \infty}$ the corresponding class of pseudodifferential operator.

By a straightforward computation (see e.g. [4]), we have $S_{h}^{m, k} \subset S_{h}^{m^{\prime}, k^{\prime}}$ iff $m \leq m^{\prime}$ and $m-\frac{h}{h+1} k \leq m^{\prime}-\frac{h}{h+1} k^{\prime} . S_{h}^{m, k}$ can be embedded in the Hörmander classes $S_{\rho, \delta}^{m+\frac{h}{h+1} k_{-}}$, where $k_{-}=\max \{0,-k\}, \rho=\delta=1 /(h+1) \leq 1 / 2$. Thus, from (5.1) we easily see that $G \in O P S_{h}^{2,2}\left(\mathbb{R}^{2}, \Sigma\right)$. 
We shall need also the following

Definition $7.2\left([4)\right.$. Let $\Omega$ and $\Sigma$ be as above. We define the class $\mathscr{H}_{h}^{m}(\Omega, \Sigma)$ by

$$
\mathscr{H}_{h}^{m}(\Omega, \Sigma)=\bigcap_{j=1}^{\infty} S_{h}^{m-j,-\frac{h+1}{h} j}(\Omega, \Sigma) .
$$

We write $\mathscr{H}_{h}^{m}$ for $\mathscr{H}_{h}^{m}\left(\mathbb{R}^{2} \times \mathbb{R}^{2}, \Sigma\right)$.

Let $\Sigma_{1}=\pi_{x} \Sigma$ be the space projection of $\Sigma$. Then we set the following

Definition 7.3. We write $H_{h}^{m}$ for $\mathscr{H}_{h}^{m}\left(\mathbb{R}_{x, y}^{2} \times \mathbb{R}_{\eta}, \Sigma_{1}\right)$, i.e., the class of all smooth functions in $\bigcap_{j=1}^{\infty} S_{h}^{m-j,-\frac{h+1}{h} j}\left(\mathbb{R}_{x, y}^{2} \times \mathbb{R}_{\eta}, \Sigma_{1}\right)$. Here $S_{h}^{m, k}\left(\mathbb{R}_{x, y}^{2} \times \mathbb{R}_{\eta}, \Sigma_{1}\right)$ denotes the set of all smooth functions such that

$$
\left|\partial_{y}^{\alpha} \partial_{\eta}^{\beta} \partial_{x}^{\gamma} a(x, y, \eta)\right| \lesssim(1+|\eta|)^{m-\beta}\left(|x|^{h}+\frac{1}{|\eta|^{\frac{h}{h+1}}}\right)^{k-\frac{\gamma}{h}} .
$$

Define the action of a symbol $a(x, y, \eta)$ in $H_{q}^{m}$ as the map $a\left(x, y, D_{y}\right): C_{0}^{\infty}\left(\mathbb{R}_{y}\right) \longrightarrow$ $C^{\infty}\left(\mathbb{R}_{x, y}^{2}\right)$ defined by

$$
a\left(x, y, D_{y}\right) u(x, y)=(2 \pi)^{-1} \int e^{i y \eta} a(x, y, \eta) \hat{u}(\eta) d \eta
$$

Such an operator, modulo a regularizing operator (w.r.t. the $y$ variable), is called a Hermite operator, and we denote by $\mathrm{OPH}_{h}^{m}$ the corresponding class.

We also need the adjoint of the Hermite operators defined in Definition 7.3 .

Definition 7.4. Let $a \in H_{h}^{m}$. We define the map $a^{*}\left(x, y, D_{y}\right): C_{0}^{\infty}\left(\mathbb{R}_{x, y}^{2}\right) \longrightarrow$ $C^{\infty}\left(\mathbb{R}_{y}\right)$ as

$$
a^{*}\left(x, y, D_{y}\right) u(y)=(2 \pi)^{-1} \iint e^{i y \eta} \overline{a(x, y, \eta)} \hat{u}(x, \eta) d x d \eta .
$$

We denote by $O P H_{h}^{* m}$ the related set of operators.

Following is a lemma on compositions involving the two different types of Hermite operators defined above. First we give a definition of "global" homogeneity:

Definition 7.5. We say that a symbol $a(x, y, \xi, \eta)$ is globally homogeneous (abbreviated g.h.) of degree $m$, if, for $\lambda \geq 1, a\left(\lambda^{-1 /(h+1)} x, y, \lambda^{1 /(h+1)} \xi, \lambda \eta\right)=\lambda^{m} a(x, y, \xi, \eta)$. Analogously a symbol, independent of $\xi$, of the form $a(x, y, \eta)$ is said to be globally homogeneous of degree $m$ if $a\left(\lambda^{-1 /(h+1)} x, y, \lambda \eta\right)=\lambda^{m} a(x, y, \eta)$.

Let $f_{-j}(x, y, \xi, \eta) \in S_{h}^{m, k+\frac{j}{h}}, j \in \mathbb{N}$. Then there exists $f(x, y, \xi, \eta) \in S_{h}^{m, k}$ such that $f \sim \sum_{j \geq 0} f_{-j}$, i.e., $f-\sum_{j=0}^{N-1} f_{-j} \in S_{h}^{m, k+\frac{N}{h}}$; thus $f$ is defined modulo a symbol in $S_{h}^{m, \infty}=\bigcap_{s \geq 0} S_{h}^{m, s}$.

Analogously, let $f_{-j}$ be globally homogeneous of degree $m-k \frac{h}{h+1}-\frac{j}{h+1}$ and such that for every $\alpha, \beta \geq 0$ satisfies the estimates

$$
\left|\partial_{(y, \eta)}^{\gamma} \partial_{x}^{\alpha} \partial_{\xi}^{\beta} f_{-j}(x, y, \xi, \eta)\right| \lesssim\left(|\xi|+|x|^{h}+1\right)^{k-\frac{\alpha}{h}-\beta}, \quad(x, \xi) \in \mathbb{R}^{2},
$$

for $(y, \eta)$ in a compact subset of $\mathbb{R} \times \mathbb{R} \backslash 0$ and every multiindex $\gamma$. Then $f_{-j} \in$ $S_{h}^{m, k+\frac{j}{h}}$. 
Accordingly, let $\phi_{-j}(x, y, \eta) \in H_{h}^{m-\frac{j}{h+1}}$. Then there exists $\phi(x, y, \eta) \in H_{h}^{m}$ such that $\phi \sim \sum_{j \geq 0} \phi_{-j}$, i.e., $\phi-\sum_{j=0}^{N-1} \phi_{-j} \in H_{h}^{m-\frac{N}{h+1}}$, so that $\phi$ is defined modulo a regularizing symbol (w.r.t. the $y$ variable).

Similarly, let $\phi_{-j}$ be globally homogeneous of degree $m-\frac{j}{h+1}$ and such that for every $\alpha, \ell \geq 0$ satisfies the estimates

$$
\left|\partial_{(y, \eta)}^{\beta} \partial_{x}^{\alpha} \phi_{-j}(x, y, \eta)\right| \lesssim\left(|x|^{h}+1\right)^{-\ell-\frac{\alpha}{h}}, \quad x \in \mathbb{R},
$$

for $(y, \eta)$ in a compact subset of $\mathbb{R} \times \mathbb{R} \backslash 0$ and every multiindex $\beta$. Then $\phi_{-j} \in$ $H_{h}^{m-\frac{j}{h+1}}$.

As a matter of fact, in the construction in Section 5 we deal with the asymptotic series of homogeneous symbols.

Next we give a brief description of the composition of the various types of operators introduced so far.

Lemma 7.1 (15], Formula 2.4.9). Let $a \in S_{h}^{m, k}, b \in S_{h}^{m^{\prime}, k^{\prime}}$, with asymptotic globally homogeneous expansions

$$
\begin{aligned}
a & \sim \sum_{j \geq 0} a_{-j}, \quad a_{-j} \in S_{h}^{m, k+\frac{j}{h}}, \text { g.h. of degree } m-\frac{h}{h+1} k-\frac{j}{h+1}, \\
b & \sim \sum_{i \geq 0} b_{-i}, \quad b_{-i} \in S_{h}^{m^{\prime}, k^{\prime}+\frac{i}{h}}, \text { g.h. of degree } m^{\prime}-\frac{h}{h+1} k^{\prime}-\frac{i}{h+1} .
\end{aligned}
$$

Then $a \circ b$ is an operator in $O P S_{h}^{m+m^{\prime}, k+k^{\prime}}$ with

$$
\begin{array}{r}
\sigma(a \circ b)-\sum_{s=0}^{N-1} \sum_{(h+1) \alpha+i+j=s} \frac{1}{\alpha !} \sigma\left(\partial_{\eta}^{\alpha} a_{-j}\left(x, y, D_{x}, \eta\right) \circ_{x} D_{y}^{\alpha} b_{-i}\left(x, y, D_{x}, \eta\right)\right) \\
\in S_{h}^{m+m^{\prime}-N, k+k^{\prime}} .
\end{array}
$$

Here $o_{x}$ denotes the composition w.r.t. the $x$-variable.

Lemma 7.2 ([4], Section 5 and [15], Sections 2.2, 2.3). Let $a \in H_{h}^{m}, b \in H_{h}^{m^{\prime}}$ and $\lambda \in S_{1,0}^{m^{\prime \prime}}\left(\mathbb{R}_{y} \times \mathbb{R}_{\eta}\right)$ with homogeneous asymptotic expansions

$$
\begin{array}{rlrl}
a & \sim \sum_{j \geq 0} a_{-j}, & a_{-j} \in H_{h}^{m-\frac{j}{h+1}}, \text { g.h. of degree } m-\frac{j}{h+1}, \\
b & \sim \sum_{i \geq 0} b_{-i}, \quad b_{-i} \in H_{h}^{m^{\prime}-\frac{i}{h+1}}, \text { g.h. of degree } m^{\prime}-\frac{i}{h+1}, \\
\lambda & \sim \sum_{\ell \geq 0} \lambda_{-\ell}, & \lambda_{-\ell} \in S_{1,0}^{m^{\prime \prime}-\frac{\ell}{h+1}}, \text { homogeneous of degree } m^{\prime \prime}-\frac{\ell}{h+1} .
\end{array}
$$

Then

(i) $a \circ b^{*}$ is an operator in $O P \mathscr{H}_{h}^{m+m^{\prime}-\frac{1}{h+1}}\left(\mathbb{R}^{2}, \Sigma\right)$ with

$$
\begin{array}{r}
\sigma\left(a \circ b^{*}\right)(x, y, \xi, \eta)-e^{-i x \xi} \sum_{s=0}^{N-1} \sum_{(h+1) \alpha+i+j=s} \frac{1}{\alpha !} \partial_{\eta}^{\alpha} a_{-j}(x, y, \eta) D_{y}^{\alpha} \hat{\bar{b}}_{-i}(\xi, y, \eta) \\
\in \mathscr{H}_{h}^{m+m^{\prime}-\frac{1}{h+1}-\frac{N}{h+1}}
\end{array}
$$

where the Fourier transform in $D_{y}^{\alpha} \hat{\bar{b}}_{-i}(\xi, y, \eta)$ is taken w.r.t. the x-variable. 
(ii) $b^{*} \circ a$ is an operator in $O P S_{1,0}^{m+m^{\prime}-\frac{1}{h+1}}\left(\mathbb{R}_{y}\right)$ with

$$
\begin{array}{r}
\sigma\left(b^{*} \circ a\right)(y, \eta)-\sum_{s=0}^{N-1} \sum_{(h+1) \alpha+j+i=s} \frac{1}{\alpha !} \int \partial_{\eta}^{\alpha} \bar{b}_{-i}(x, y, \eta) D_{y}^{\alpha} a_{-j}(x, y, \eta) d x \\
\in S_{1,0}^{m+m^{\prime}-\frac{1}{h+1}-\frac{N}{h+1}}\left(\mathbb{R}_{y} \times \mathbb{R}_{\eta}\right) .
\end{array}
$$

(iii) $a \circ \lambda$ is an operator in $O P H_{h}^{m+m^{\prime \prime}}$. Furthermore its asymptotic expansion is given by

$$
\sigma(a \circ \lambda)-\sum_{s=0}^{N-1} \sum_{(h+1) \alpha+j+\ell=s} \frac{1}{\alpha !} \partial_{\eta}^{\alpha} a_{-j}(x, y, \eta) D_{y}^{\alpha} \lambda_{-\ell}(y, \eta) \in H_{h}^{m+m^{\prime \prime}-\frac{N}{h+1}} .
$$

Lemma 7.3. Let $a\left(x, y, D_{x}, D_{y}\right)$ be an operator in the class $O P S_{h}^{m, k}\left(\mathbb{R}^{2}, \Sigma\right)$ and $b\left(x, y, D_{y}\right) \in O P H_{h}^{m^{\prime}}$ with g.h. asymptotic expansions

$$
\begin{aligned}
a & \sim \sum_{j \geq 0} a_{-j}, \quad a_{-j} \in S_{h}^{m, k+\frac{j}{h}}, \text { g.h. of degree } m-\frac{h}{h+1} k-\frac{j}{h+1}, \\
b & \sim \sum_{i \geq 0} b_{-i}, \quad b_{-i} \in H_{h}^{m^{\prime}-\frac{i}{h}}, \text { g.h. of degree } m^{\prime}-\frac{i}{h+1} .
\end{aligned}
$$

Then $a \circ b \in O P H_{h}^{m+m^{\prime}-k \frac{h}{h+1}}$ and has a g.h. asymptotic expansion of the form

$$
\begin{aligned}
& \sigma(a \circ b)-\sum_{s=0}^{N-1} \sum_{(h+1) \ell+i+j=s} \frac{1}{\ell !} \partial_{\eta}^{\ell} a_{-j}\left(x, y, D_{x}, \eta\right)\left(D_{y}^{\ell} b_{-i}(\cdot, y, \eta)\right) \\
& \in H_{h}^{m+m^{\prime}-k \frac{h}{h+1}-\frac{N}{h+1}}
\end{aligned}
$$

Lemma 7.4. Let $a\left(x, y, D_{x}, D_{y}\right)$ be an operator in the class $O P S_{h}^{m, k}\left(\mathbb{R}^{2}, \Sigma\right)$, $b^{*}\left(x, y, D_{y}\right) \in O P H_{h}^{* m^{\prime}}$ and $\lambda\left(y, D_{y}\right) \in O P S_{1,0}^{m^{\prime \prime}}\left(\mathbb{R}_{y}\right)$ with homogeneous asymptotic expansions

$$
\begin{aligned}
a & \sim \sum_{j \geq 0} a_{-j}, & a_{-j} \in S_{y}^{m, k+\frac{j}{h}}, \text { g.h. of degree } m-\frac{h}{h+1} k-\frac{j}{h+1}, \\
b & \sim \sum_{i \geq 0} b_{-i}, & b_{-i} \in H_{h}^{m^{\prime}-\frac{i}{h}}, \text { g.h. of degree } m^{\prime}-\frac{i}{h+1}, \\
\lambda & \sim \sum_{\ell \geq 0} \lambda_{-\ell}, & \lambda_{-\ell} \in S_{1,0}^{m^{\prime \prime}-\frac{\ell}{h+1}}, \text { homogeneous of degree } m^{\prime \prime}-\frac{\ell}{h+1} .
\end{aligned}
$$

Then

(i) $b^{*}\left(x, y, D_{y}\right) \circ a\left(x, y, D_{x}, D_{y}\right) \in O P H_{h}^{* m+m^{\prime}-\frac{h}{h+1} k}$ with g.h. asymptotic expansion

$$
\begin{array}{r}
\sigma\left(b^{*} \circ a\right)-\sum_{s=0}^{N-1} \sum_{(h+1) \ell+i+j=s} \frac{1}{\ell !} D_{y}^{\ell}\left(\overline{a_{-j}}\left(x, y, D_{x}, \eta\right)\right)^{*}\left(\partial_{\eta}^{\ell} \overline{b_{-i}}(\cdot, y, \eta)\right) \\
\in H_{h}{ }^{m+m^{\prime}-k \frac{h}{h+1}-\frac{N}{h+1}}
\end{array}
$$


(ii) $\lambda\left(y, D_{y}\right) \circ b^{*}\left(x, y, D_{y}\right) \in O P H_{h}^{* m^{\prime}+m^{\prime \prime}}$ with asymptotic expansion

$$
\sigma\left(\lambda \circ b^{*}\right)-\sum_{s=0}^{N-1} \sum_{(h+1) \alpha+i+\ell=s} \frac{1}{\alpha !} \partial_{\eta}^{\alpha} \lambda_{-\ell}(y, \eta) D_{y}^{\alpha} \overline{b_{-i}}(x, y, \eta) \in H_{h}^{m^{\prime}+m^{\prime \prime}-\frac{N}{h+1}} .
$$

Finally, let us conclude this appendix by stating (without proving) the Sobolev continuity properties of the operators defined above.

Lemma 7.5. (a) Let $a \in S_{h}^{m, k}$, properly supported, with $k \leq 0$. Then Op $a$ is continuous from $H_{\text {loc }}^{s}\left(\mathbb{R}^{2}\right)$ to $H_{\text {loc }}^{s-m+k \frac{h}{h+1}}\left(\mathbb{R}^{2}\right)$.

(b) Let $\phi \in H_{h}^{m+\frac{1}{2(h+1)}}$, properly supported. Then Op $\phi$ is continuous from $H_{\text {loc }}^{s}(\mathbb{R})$ to $H_{\text {loc }}^{s-m}\left(\mathbb{R}^{2}\right)$. Moreover, $\phi^{*}\left(x, y, D_{y}\right)$ is continuous from $H_{\text {loc }}^{s}\left(\mathbb{R}^{2}\right)$ to $H_{\text {loc }}^{s-m}(\mathbb{R})$.

\section{ACKNOWLEDGMENTS}

The author wishes to thank Bernard Helffer for helpful discussions and, in particular, for suggesting the improvements in Section 3. The author is also grateful to Alexander V. Sobolev for pointing out the existence of sharp upper bounds for the number of negative eigenvalues of $P_{\mu}$.

\section{REFERENCES}

[1] F. A. Berezin and M. A. Shubin, The Schrödinger equation, Mathematics and its Applications (Soviet Series), vol. 66, Kluwer Academic Publishers Group, Dordrecht, 1991. Translated from the 1983 Russian edition by Yu. Rajabov, D. A. Leĭtes and N. A. Sakharova and revised by Shubin. With contributions by G. L. Litvinov and Leŭtes. MR 1186643 (93i:81001)

[2] P. Bolley, J. Camus, and B. Helffer, Sur une classe d'operateurs partiellement hypoelliptiques, J. Math. Pures Appl. (9) 55 (1976), no. 2, 131-171. MR0427808 (55 \#838)

[3] Louis Boutet de Monvel and François Trèves, On a class of pseudodifferential operators with double characteristics, Invent. Math. 24 (1974), 1-34. MR0353064 (50 \#5550)

[4] Louis Boutet de Monvel, Hypoelliptic operators with double characteristics and related pseudo-differential operators, Comm. Pure Appl. Math. 27 (1974), 585-639. MR0370271 (51 \#6498)

[5] Antonio Bove and Marco Mughetti, Analytic and Gevrey hypoellipticity for a class of pseudodifferential operators in one variable, J. Differential Equations 255 (2013), no. 4, 728-758, DOI 10.1016/j.jde.2013.04.030. MR3056589

[6] Antonio Bove, Marco Mughetti, and David S. Tartakoff, Gevrey hypoellipticity for an interesting variant of Kohn's operator, Complex analysis, Trends Math., Birkhäuser/Springer Basel AG, Basel, 2010, pp. 51-73, DOI 10.1007/978-3-0346-0009-5_3. MR2885108 (2012k:35067)

[7] Antonio Bove, Marco Mughetti, and David S. Tartakoff, Hypoellipticity and nonhypoellipticity for sums of squares of complex vector fields, Anal. PDE 6 (2013), no. 2, 371-445, DOI 10.2140/apde.2013.6.371. MR3071394

[8] K. Chadan, N. N. Khuri, A. Martin, and Tai Tsun Wu, Bound states in one and two spatial dimensions, J. Math. Phys. 44 (2003), no. 2, 406-422, DOI 10.1063/1.1532538. MR.1952194 (2005f:81273)

[9] Paulo D. Cordaro and Nicholas Hanges, Impact of lower order terms on a model $P D E$ in two variables, Geometric analysis of PDE and several complex variables, Contemp. Math., vol. 368, Amer. Math. Soc., Providence, RI, 2005, pp. 157-176, DOI 10.1090/conm/368/06777. MR2126468(2005j:35024)

[10] C. Fefferman and D. H. Phong, On positivity of pseudo-differential operators, Proc. Nat. Acad. Sci. U.S.A. 75 (1978), no. 10, 4673-4674. MR.507931 (80b:47064)

[11] Antonio Gilioli and François Trèves, An example in the solvability theory of linear PDE's, Amer. J. Math. 96 (1974), 367-385. MR0355285 (50 \#7761) 
[12] Antonio Gilioli, A class of second-order evolution equations with double characteristics, Ann. Scuola Norm. Sup. Pisa Cl. Sci. (4) 3 (1976), no. 2, 187-229. MR0437961 (55 \#10882)

[13] V.V. Grushin, On a class of elliptic pseudodifferential operators degenerate on a submanifold, Mat. Sbornik 84(126) n.2 (1971), 155-185.

[14] Nicholas Hanges and A. Alexandrou Himonas, Singular solutions for a class of Grusin type operators, Proc. Amer. Math. Soc. 124 (1996), no. 5, 1549-1557, DOI 10.1090/S0002-993996-03180-2. MR1307525 (96g:35035)

[15] Bernard Helffer, Sur l'hypoellipticité des opérateurs pseudodifferentiels à caractéristiques multiples (perte de 3/2 dérivées) (French), Bull. Soc. Math. France Suppl. Mém. 51-52 (1977), 13-61. MR0460864 (57 \#855)

[16] Bernard Helffer, Théorie spectrale pour des opérateurs globalement elliptiques (French), Astérisque, vol. 112, Société Mathématique de France, Paris, 1984. With an English summary. MR743094 (86d:35151)

[17] B. Helffer and J. Sjöstrand, Multiple wells in the semiclassical limit. I, Comm. Partial Differential Equations 9 (1984), no. 4, 337-408, DOI 10.1080/03605308408820335. MR740094 (86c:35113)

[18] B. Helffer, The Montgomery model revisited, Colloq. Math. 118 (2010), no. 2, 391-400, DOI 10.4064/cm118-2-3. MR2602157 (2011c:35377)

[19] B. Helffer and M. Persson, Spectral properties of higher order anharmonic oscillators, J. Math. Sci. (N. Y.) 165 (2010), no. 1, 110-126, DOI 10.1007/s10958-010-9784-5. Problems in mathematical analysis. No. 44. MR $2838999(2012 \mathrm{~m}: 34155)$

[20] Lars Hörmander, Pseudo-differential operators and non-elliptic boundary problems, Ann. of Math. (2) 83 (1966), 129-209. MR0233064 (38 \#1387)

[21] Lars Hörmander, The Cauchy problem for differential equations with double characteristics, J. Analyse Math. 32 (1977), 118-196. MR0492751 (58 \#11822)

[22] L. Hörmander, The Analysis of Linear Partial Differential Operators, Vol. III-IV, SpringerVerlag (1985). MR0781536 MR0781537

[23] Tosio Kato, Perturbation theory for linear operators, Die Grundlehren der mathematischen Wissenschaften, Band 132, Springer-Verlag New York, Inc., New York, 1966. MR0203473 (34 \#3324)

[24] J. J. Kohn, Hypoellipticity and loss of derivatives, Ann. of Math. (2) 162 (2005), no. 2, 943 986, DOI 10.4007/annals.2005.162.943. With an appendix by Makhlouf Derridj and David S. Tartakoff. MR2183286 (2006k:35036)

[25] André Martinez and Michel Rouleux, Effet tunnel entre puits dégénérés (French), Comm. Partial Differential Equations 13 (1988), no. 9, 1157-1187, DOI 10.1080/03605308808820571. MR.946285 (89h:35085)

[26] A. Menikoff, Some examples of hypoelliptic partial differential equations, Math. Ann. 221 (1976), no. 2, 167-181. MR0481452 (58 \#1568)

[27] Marco Mughetti, A problem of transversal anisotropic ellipticity, Rend. Sem. Mat. Univ. Padova 106 (2001), 111-142. MR.1876216 (2003a:35217)

[28] Marco Mughetti, Hypoellipticity and higher order Levi conditions, J. Differential Equations 257 (2014), no. 4, 1246-1287, DOI 10.1016/j.jde.2014.05.008. MR.3210028

[29] Marco Mughetti, Regularity properties of a double characteristics differential operator with complex lower order terms, J. Pseudo-Differ. Oper. Appl. 5 (2014), no. 3, 343-358, DOI 10.1007/s11868-014-0093-5. MR.3249940

[30] Cesare Parenti and Alberto Parmeggiani, On the hypoellipticity with a big loss of derivatives, Kyushu J. Math. 59 (2005), no. 1, 155-230, DOI 10.2206/kyushujm.59.155. MR2134059 (2005m:35049)

[31] Marco Mughetti, Cesare Parenti, and Alberto Parmeggiani, Lower bound estimates without transversal ellipticity, Comm. Partial Differential Equations 32 (2007), no. 7-9, 1399-1438, DOI 10.1080/03605300600962671. MR2354498 (2009b:35454)

[32] Michael Reed and Barry Simon, Methods of modern mathematical physics. IV. Analysis of operators, Academic Press [Harcourt Brace Jovanovich Publishers], New York, 1978. MR0493421 (58 \#12429c)

[33] Barry Simon, Coupling constant analyticity for the anharmonic oscillator. (With appendix), Ann. Physics 58 (1970), 76-136. MR0416322 (54 \#4397) 
[34] Barry Simon, Semiclassical analysis of low lying eigenvalues. I. Nondegenerate minima: asymptotic expansions (English, with French summary), Ann. Inst. H. Poincaré Sect. A (N.S.) 38 (1983), no. 3, 295-308. MR708966 (85m:81040a)

[35] Johannes Sjöstrand, Parametrices for pseudodifferential operators with multiple characteristics, Ark. Mat. 12 (1974), 85-130. MR0352749 (50 \#5236)

[36] Johannes Sjöstrand and Maciej Zworski, Elementary linear algebra for advanced spectral problems (English, with English and French summaries), Ann. Inst. Fourier (Grenoble) 57 (2007), no. 7, 2095-2141. Festival Yves Colin de Verdière. MR2394537 (2009m:35351)

Department of Mathematics, University of Bologna, Piazza di Porta S. Donato 5, 40127 Bologna, ITALY

E-mail address: mughetti@dm.unibo.it 